University of Arkansas, Fayetteville

ScholarWorks@UARK

Graduate Theses and Dissertations

$7-2015$

\title{
Adaptive Controller Using Runtime Partial Hardware Reconfiguration for Unmanned Aerial Vehicles (UAVs)
}

Nikhil Thomas

University of Arkansas, Fayetteville

Follow this and additional works at: https://scholarworks.uark.edu/etd

Part of the Other Computer Engineering Commons

\section{Citation}

Thomas, N. (2015). Adaptive Controller Using Runtime Partial Hardware Reconfiguration for Unmanned Aerial Vehicles (UAVs). Graduate Theses and Dissertations Retrieved from https://scholarworks.uark.edu/ etd/1254

This Thesis is brought to you for free and open access by ScholarWorks@UARK. It has been accepted for inclusion in Graduate Theses and Dissertations by an authorized administrator of ScholarWorks@UARK. For more information, please contact scholar@uark.edu. 
Adaptive Controller Using Runtime Partial Hardware Reconfiguration for Unmanned Aerial Vehicles (UAVs)

A thesis submitted in partial fulfillment

of the requirements for the degree of

Master of Science in Computer Engineering

by

\author{
Nikhil Thomas \\ Cochin University of Science and Technology \\ Bachelor of Technology in Computer Science and Computer Engineering, 2009 \\ University of Arkansas \\ Master of Science in Microelectronics-Photonics, 2013
}

July 2015

University of Arkansas

This thesis is approved for the recommendation of the Graduate Council.

Dr. Christophe Bobda

Thesis Director

Dr. David Andrews

Dr. James Parkerson

Committee Member

Committee Member

Dr. Dharmendra Saraswat

Co-Advisor 


\begin{abstract}
The goal of this thesis is to explore the feasibility of a multirotor controller system which can dynamically change the arm configuration of a multirotor. Currently most of the multirotor systems have to be powered down, rewired, and programmed with new firmware, to configure how many arms/motors they use to fly. The focus of our effort is to develop a Field Programmable Gate Array (FPGA) based hardware/software controller which uses dynamic partial hardware reconfiguration to switch the arm/motor configuration of a multirotor during operation. We believe that this will make a multirotor more fault tolerant and adaptive. This thesis explains the design and development of a simple multirotor reconfiguration system which can start with a six arm configuration and switch to a four arm configuration or a three arm configuration and back.

The design and implementation of the hardware and software on a Xilinx Zynq System on chip prototyping board (ZedBoard) is explained in this thesis. The system is used for single axis balancing of the multirotor. Observations from the experiments, limitations of the current design, and potential future developments are also listed in this thesis.
\end{abstract}




\section{Acknowledgement}

I express my gratitude to Dr. Christophe Bobda and Dr. Dharmendra Saraswat for trusting me. I thank you both for giving me this opportunity to work on the projects you assigned me. My experience as a graduate student has helped develop myself as a skilled engineer and as

a disciplined person. Thank you for pushing me hard. Thank you for making me realize that I can work harder than what I used to believe is my limit.

I thank my committee members Dr. David Andrews and Dr. James Parkerson for their support in my work for this thesis project. I will always be grateful to everyone who has helped me learn and improve my skills.

I thank all my colleagues who always helped me progress in my work. I thank Ben Hancock for giving me a strong foundation. I appreciate your patience and your time. I thank Luca Bochi Saldanha, Franck Yonga, and Michael Mefenza for motivating me to work hard and for appreciating my efforts. I also thank Danny Thepvongsa and Andrew Felder for their effort in accelerating the progress of this project.

I thank my parents for giving me good education and loving me unconditionally. I also thank my brother for always being there for me.

And I thank my partner Seena Solomon, for making me smile, making me hopeful, making me pray and motivating me to be good a person. 


\section{Dedication}

I dedicate this work to God, the master of all creativity. 
Table of Contents

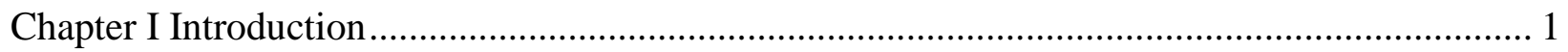

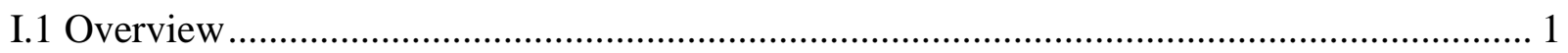

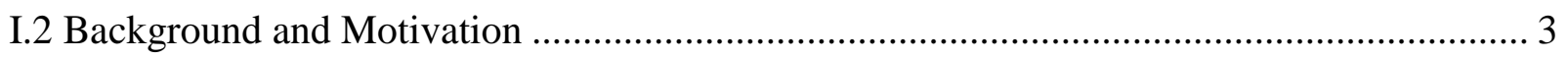

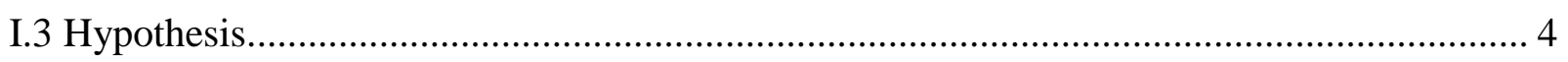

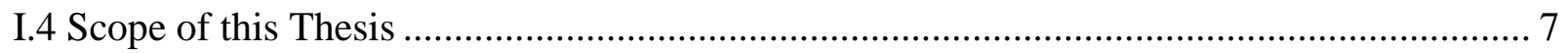

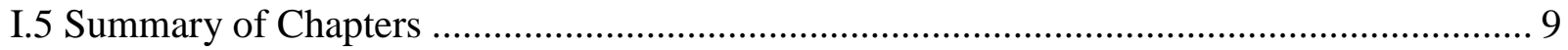

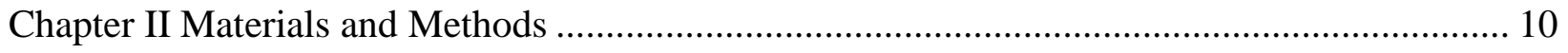

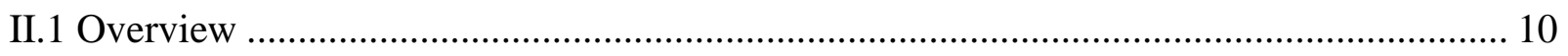

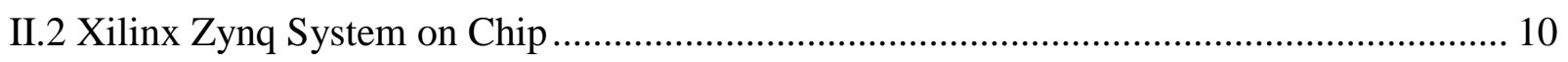

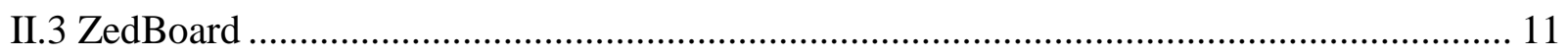

II.4 Dynamic Partial Hardware Reconfiguration ......................................................... 13

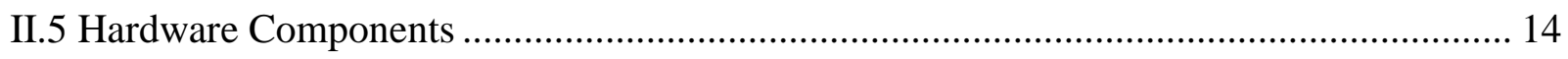

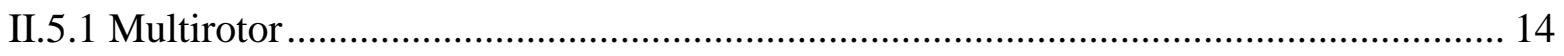

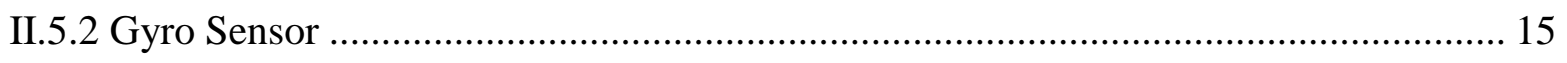

II.5.3 Voltage and Current Sensor ........................................................................ 16

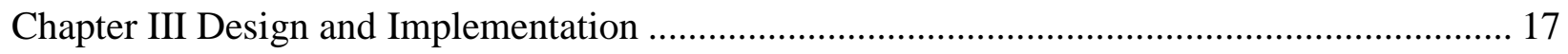

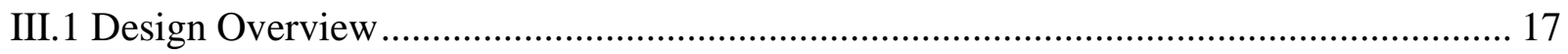

III.2 Multirotor Configurations Considered in this Project ................................................ 19

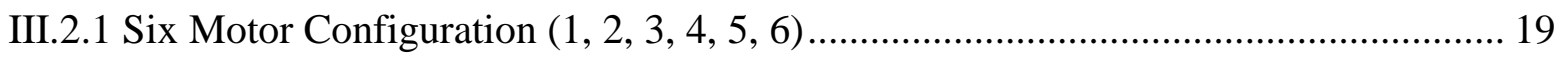

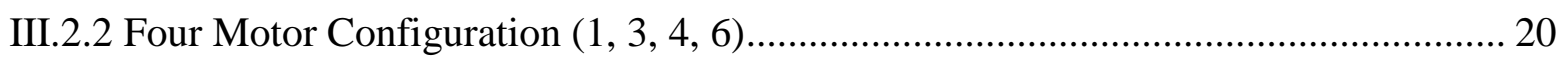

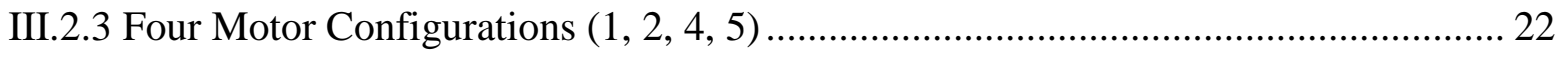

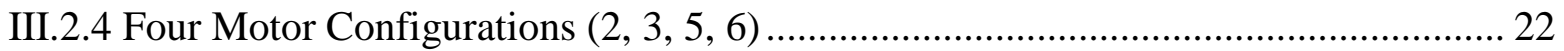

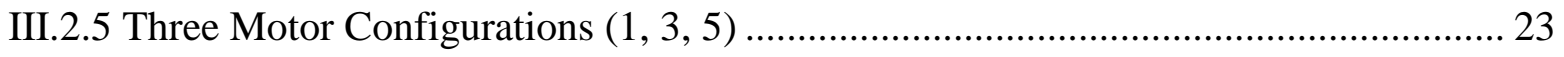

III.2.6 Three Motor Configurations $(2,4,6)$................................................................. 24

III.3 Control Module Switching Logic ........................................................................... 25

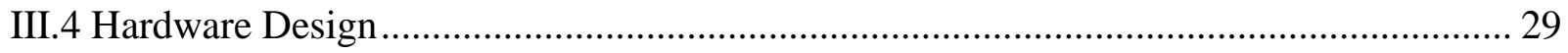

III.4.1 Design0: Simple Design without Partial Hardware Reconfiguration........................ 29

III.4.2 Design1: Hardware Design with a Reconfigurable Partition .................................. 30

III.4.3 Design2: Prototype with PID and Gyro sensor .............................................. 31

III.4.4 Design of Individual Control Modules ............................................................ 33

III.4.5 Design of PWM Generator ............................................................................... 34 


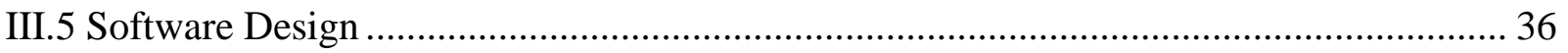

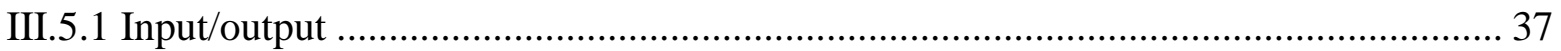

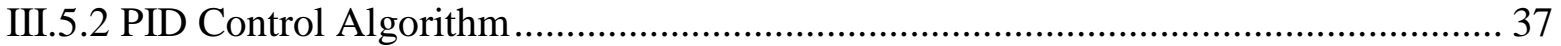

III.5.3 Managing Partial Hardware Reconfiguration ............................................................. 37

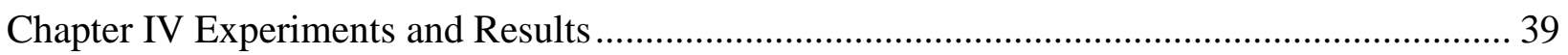

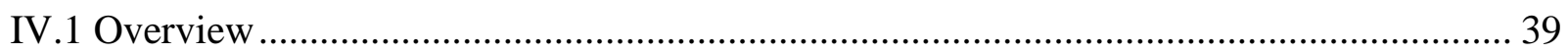

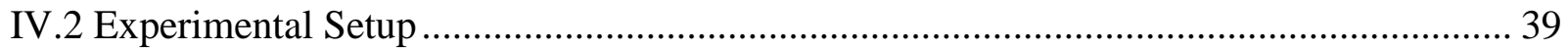

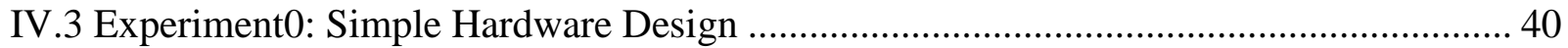

IV.4 Experiment1: Hardware Design with a Reconfigurable Partition .................................... 41

IV.5 Experiment2: Prototype with PID control ................................................................. 43

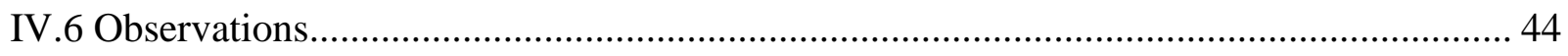

IV.6.1 PWM vs Current............................................................................................. 44

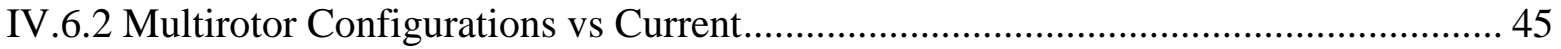

Chapter V Conclusion and Future Work .................................................................................. 47

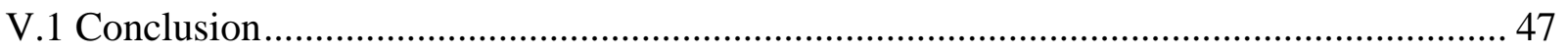

V.2 Potential Applications ………………………….................................................... 49

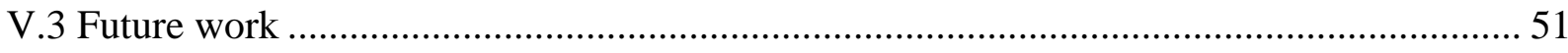

V.3.1 Multiplexed Dual Reconfigurable Partitions ............................................................. 51

V.3.2 User Input from a Radio/Bluetooth Controller .......................................................... 52

V.3.3 Mathematical Modeling of Multirotor ................................................................... 52

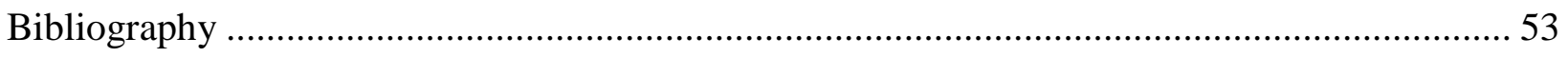




\section{List of Figures}

Figure I.1: Expected variation in load carrying capacity and duration of flight of different multirotor flight configurations when the motors, propellers and the battery is of the same type. 4 Figure II.1: Zynq Processing System (PS) and Programmable Logic (PL)................................ 11

Figure II.2: ZedBoard (Photo taken by author) ……………………………………............. 12

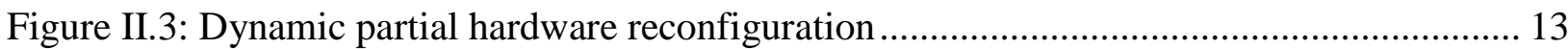

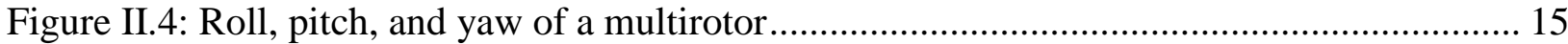

Figure II.5: Digilent PmodGYRO 3-Axis digital gyroscope (Photo taken by author) ................. 16

Figure II.6: Sparkfun AttoPilot voltage and current sensor (Photo taken by author) .................... 16

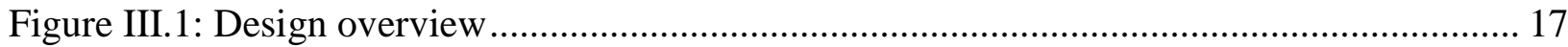

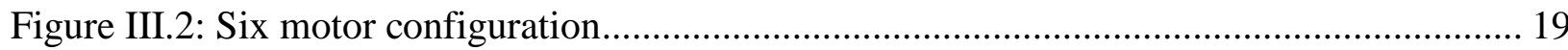

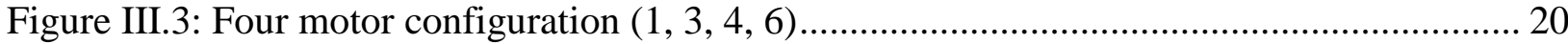

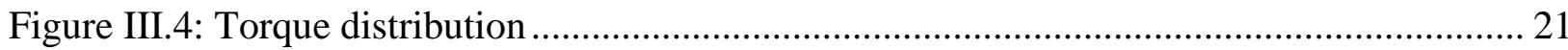

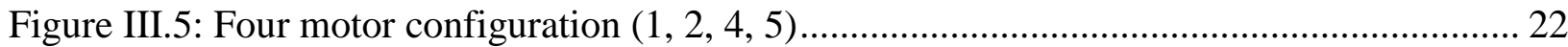

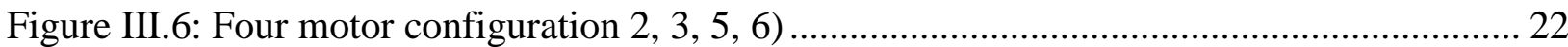

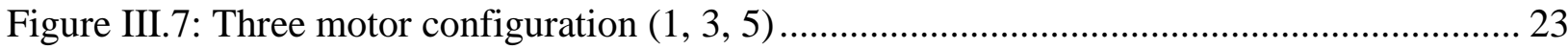

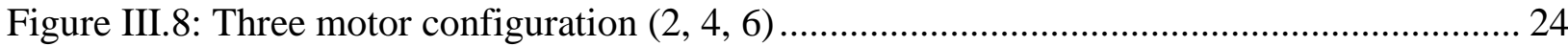

Figure III.9: A valid configuration (left), an invalid configuration (right) .................................. 27

Figure III.10: Hardware design without partial reconfiguration. Flat design: all seven configurations are connected in through a 7-to-1 multiplexer to the output.................................. 29 Figure III.11: Hardware design with a reconfigurable partition, seven different configuration are

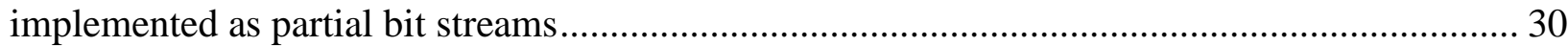

Figure III.12: Gyro sensor and timer added to hardware design which already has a

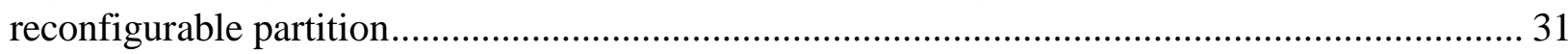

Figure III.13: Structure of a reconfigurable control module 'four motor 1 ' $(1,3,4,6)$................ 34

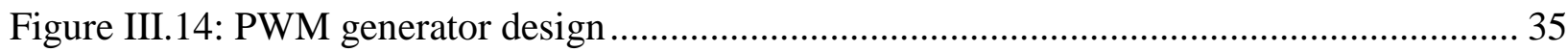

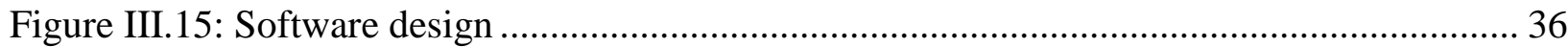

Figure IV.1: Experimental setup (Photo taken by author) ............................................................. 39

Figure IV.2: PWM duty vs Current (at different values of Voltage) ........................................... 45

Figure IV.3: Multirotor configuration vs Current (at different values of Voltage) ...................... 46 


\section{List of Tables}

Table III.1: Multirotor control switching logic input and output configurations ....................... 26

Table III.2: Mapping of input switch values to a multirotor arm configuration........................ 28

Table III.3: PWM minimum duty and maximum duty for different period values .................... 36

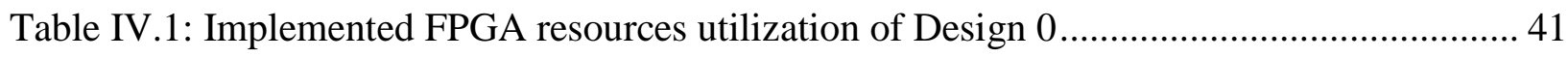

Table IV.2: Implemented FPGA resources utilization of Design 1 ...................................... 42

Table IV.3: Implemented FPGA resources utilization of Design 2 ...................................... 43

Table IV.4: Current drawn at different PWM duty (higher the PWM higher the motor speed) .. 44

Table IV.5: Current drawn for different configurations (more motor draw more current) ......... 45

Table V.1: Theoretical estimation of operation time ....................................................... 49

Table V.2: Hypothetical application: table showing operation duration for different configurations for a given load 


\section{Chapter I Introduction}

\section{I.1 Overview}

A multirotor, or a multicopter, is an aircraft which uses more than one motorized propeller to propel itself. A multirotor is aerodynamically unstable, which means it needs an intelligent controller to maintain a stable state while in operation. Unlike a fixed wing aircraft a multirotor cannot glide and land when the controller malfunctions or when the propulsion goes offline. The main advantage of multirotors over fixed wing aircraft is their ability to vertically takeoff and land (VTOL). Hence multirotors do not need a runway for takeoff or landing, making them ideal for geographically challenging areas. Nowadays, multirotors are used in various areas such as aerial photography, geospatial studies, etc [1]. Multirotor technology developed so fast from being a toy to a highly useful tool which has applications in both scientific research and entertainment.

The advancements in components and materials has made multirotor more efficient. Brushless motors and Lithium Polymer (LiPo) batteries are key factors in the advancement of multirotor technology $[2,3]$. Development in multirotor control software equipped with features like autonomous navigation, has made multirotors more intelligent. Cutting edge research is taking place in design, development, and applications of multirotors. Various onboard image processing technologies help multirotors collect and process visual inputs $[4,5]$. On the one hand, tiny multirotors can communicate with each other and collaborate in performing a task [6]. On the other, heavy duty multirotors are being developed to retrieve and deliver packages [7]. Multirotors which can be used for human transportation vehicles are also in development $[8,9]$.

A variety of excellent off-the-shelf flight controller boards are now available. The flight control boards are mass produced hardware and the opportunity to customize and develop the 
hardware is less compared to customizability in flight control software. Off-the-shelf flight control boards abstract their internal design behind their hardware abstraction layers (HAL). Hence it becomes very difficult for an interested user to learn multirotor techniques implemented beneath the HAL. An aspect of multirotor technology which is not explored a lot is the customization of flight control hardware circuit.

We believe that the rapidly developing computer hardware technology Field Programmable Gate Arrays (FPGAs) can be used to address this problem. The goals of this thesis are

- To experiment with FPGA based multirotor control hardware

- To make adaptive multirotor controllers using runtime partial hardware reconfiguration of FPGAs technology

- To investigate advantages of using a FPGA based multirotor controller The main advantage of FPGAs is that they are programmable hardware [10]. That is, a FPGA is a fabric of logic units which can be used to rapidly prototype hardware designs developed in Hardware Description Languages (HDL) such as VHDL, Verilog, etc. Efficient hardware can be developed using FPGA technology. The possibility to customize the hardware is endless. The most satisfying aspect in the development of FPGA based multirotor controller is the combined hardware/software experience. The developer gets complete access to all hardware and software features of the system. However, FPGA based flight controllers are in their initial stages of development. There is a lot more work to be done in this direction to make top level software features of off-the-shelf multirotor control boards available with FPGA based systems. 


\section{I.2 Background and Motivation}

Multirotor controllers started as simple systems which control motor rotation speed with respect to the input from an inertial measurement unit (IMU). Now there are low cost off-theshelf multirotor flight control boards like APM2.x, Pixhawk, Hoverfly Open etc. which can precisely control all aspects of multirotor flight as well as interact with other peripherals like cameras, navigate autonomously, stream live video etc. Depending upon the number of motors in a multirotor setup different flight configurations are possible such as Quad, Hex, and Octo etc [11]. Almost all of the flight control boards support most of the common multirotor flight configurations. Normally none of them can change the flight configuration while in operation. The aircraft has to land, the motors have to be connected to different ports of the board and a different flight configuration will need a different firmware. The technological possibility of developing a hardware/software flight control system which can change the flight configuration of a copter while in flight needs to be explored.

All components of a multirotor have to be designed carefully and fine-tuned to precisely control all physical forces acting upon the multirotor so that we can maneuver the aircraft exactly the way we want. We acknowledge the fact that altering any physical, software or hardware parameters of a multirotor while in flight can make it unstable and produce disastrous results. Therefore we want to capitalize on our experience with FPGA prototyping and hardware/software co-design to come up with an adaptive multirotor flight control system which can switch between different multirotor arm configurations. The technology runtime partial hardware reconfiguration of FPGAs allows us to change a part of a hardware design implemented on a FPGA fabric during runtime $[12,13]$. Moreover, recent developments have shown that modern technologies like 'Xilinx Zynq System on Chip' which combine a hard 
processing element with a FPGA fabric has immense potential in the domain of multirotor flight control [4].

\section{I.3 Hypothesis}

The load carrying capacity of a multirotor depends on the number of motors it has, if we are comparing different flight configurations with the same type of motors and propellers. A multirotor draws power for its operation from a LiPo battery. The more powerful the battery, the longer the flight duration of the multirotor. As the number of motors increase the more power the multirotor draws from the battery. Hence, increase in the number of motors will increase the load carrying capacity but it will reduce flight duration when the same type of battery is used (Figure I.1). This is a simplistic model within the scope of this work. In the future developments more rigorous analysis need to be done to make accurate estimation of load and flight duration [14].

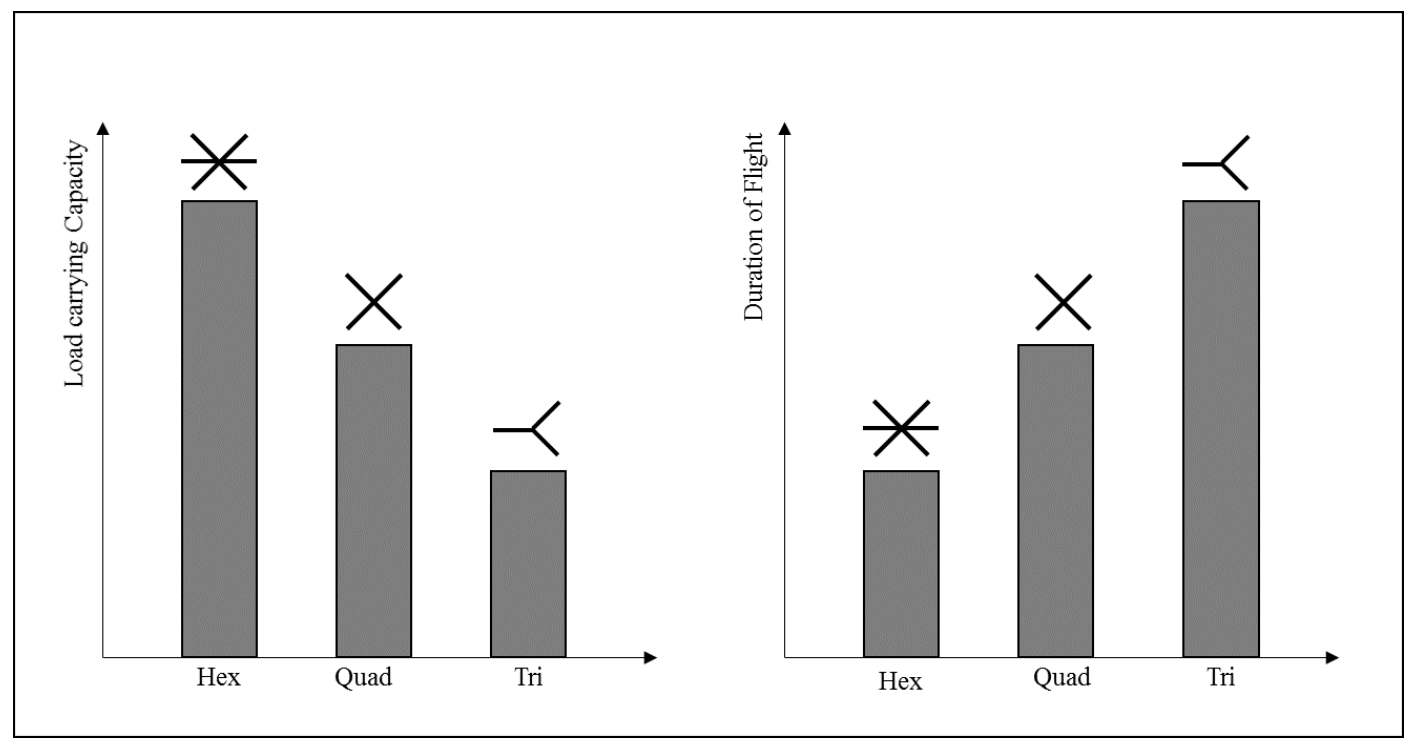

Figure I.1: Expected variation in load carrying capacity and duration of flight of different multirotor flight configurations when the motors, propellers and the battery is of the same type

Our hypothesis is that by making use of the runtime partial hardware reconfiguration technology we can design and implement a multirotor controller which can switch its flight configuration while still in flight. Imagine a hexacopter which can switch to a quadcopter 
configuration and then to a tricopter configuration as needed. We understand the challenges with respect to balancing the physical forces on a multirotor like angular momentum. Conventionally, the total angular momentum of a multirotor is balanced by alternating the propeller spin direction (clock wise and counter clockwise) [11]. We intend to use the alternate configurations which use two motors per arm of a multirotor in the future. The upward facing motors with clockwise propellers can have clockwise spin and the downward facing motors with counter clockwise propellers can have counter clockwise spin. These configurations should make the net angular momentum of the two motors in an arm cancel out each other. Therefore if we use the configurations tricopter with six motors (Y6), quadcopter with eight motors (X8) and a hexacopter with twelve motors it should be possible to balance the net angular momentum even after dynamically switching the configurations. The double motor configurations are not implemented in this work. They needed to be tested in future developments.

Our goal is to explore the challenges in developing multirotor controllers which can accommodate the flexibility to choose a flight configuration as and when the need arises. For example a hexacopter with an adaptable flight controller can switch between different flight configurations. It can use all motors to configure itself as a hexacopter. There are three 'four motor' configurations available depending upon the motors being used. It also has two flight configurations where three motors are used to attain stable flight. We think this feature has a wide range of applications. The potential advantages of adaptive flight configuration can be summarized into two categories:

1. Fault Tolerance: If a motor in the proposed multirotor setup malfunctions, it can switch into a configuration which does not use that motor and still maintain stable flight. It can also recruit all motors to attain steady flight in adverse environmental conditions like 
strong winds. That is if strong winds are causing disturbances while the multirotor is flying with a three arm configuration, it can switch itself into a configuration that uses four or six arms. Hence a multirotor which can change its flight configuration during operation will be more fault tolerant.

2. Optimized Performance: The required thrust, sturdiness of flight, flight duration, and speed can vary depending upon the factors such as load, environmental condition, time and power constraints etc. A multirotor with runtime arm configuration adaptivity will give the user the ability to control power utilization and flight duration. Imagine a multirotor used for delivering a relatively heavy package. The multirotor (twelve motors in hex configuration) might need to use all of its motors to carry the load to its destination. However, it might need much less load carrying capacity to fly back to its base. An operator can choose the flight configurations depending upon the nature of the flight mission. This scenario has a lot of relevance as studies are being done on the use of aerial vehicles to deliver packages $[7,15]$.

In addition, we firmly believe that FPGAs have a major role in unifying the software hardware design process. In any computer system the limit for the performance of software is the underlying hardware. Use of FPGAs can push this limit and make system design process a simultaneous development of hardware and software components. In other words, FPGA technology offers more transparency in the hardware domain, which in turn helps us write better software with functions abstracted in both hardware and software modules.

Advanced features like runtime partial hardware reconfiguration can lead to more powerful control boards for multirotors. Runtime partial hardware reconfiguration adds flexibility and customizability on the same amount of FPGA real estate. Functions like image 
processing for feature/object detection, telecommunication etc. can be designed as reconfigurable partitions which will lead to more powerful multirotor flight control systems. Therefore we believe that there is high potential in this research direction where FPGAs or System on Chip technology is used as a platform which not only controls multirotor flight but also supports more high level applications of multirotors.

\section{I.4 Scope of this Thesis}

This thesis is our first step in the direction of developing adaptive multirotor controller which supports adaptive flight configurations. Our goal is to design, implement, and study the performance of adaptive multirotor configurations with respect to one rotational degree of freedom, the roll (angle measure on $\mathrm{x}$ axis with respect to center of gravity of multirotor) [16]. We have a hexacopter multirotor setup which can pivot along its roll axis. The features of the multirotor and the experimental setup will be discussed in the coming chapters. We have designed a hardware software system which has a dynamically reconfigurable hardware module which can be reconfigured with modules associated with different flight configurations.

We are not trying to develop a new control algorithm for multirotor flight. Hence this thesis will not discuss mathematical modeling of multirotors. We are using a Proportional Integral Derivative (PID) control algorithm to balance the multirotor on its roll axis [17]. We have implemented the PID algorithm in the software side of the system. That is the PID algorithm runs on top of the hardware platform consisting of the Zynq processing system (Cortex A9 processor) and our custom hardware (which has a reconfigurable hardware partition).

Currently the functions of our custom hardware are reading input from a gyro sensor which measures angular velocity of the multirotor and producing pulse width modulated (PWM) signals 
to control the motor speeds. The reconfigurable hardware partition distributes the PWM signals to the motors depending on the current configuration and control signals from the software The Xilinx Zynq System on Chip prototyping board 'the ZedBoard' is used to test our design. Zynq has a processing system which consists of two Cortex A9 processors and programmable logic which is the FPGA fabric. Our hardware is designed in VHDL using hardware prototyping tools from Xilinx. The software side of our system is implemented in C. The software communicates with the custom hardware through memory mapped registers.

We used a commercially available hexacopter as our multirotor (XAircraft DIY Hexa) to test our design. The hexacopter came with a third party flight control board (APM 2.5). The flight control board was powerful and had many features. However, we wanted to use our FPGA based multirotor controller design. We connected the electronic speed controllers of the hexacopter to our hardware/software adaptive control system directly. We used a gyro sensor peripheral module from Digilent Inc to measure the angular velocity of the multirotor in the three axis. We started with a simple design then iteratively modified it. The development of this base system went through three design iterations.

1. Adaptive configuration controller without partial hardware reconfiguration: This design consists of all the different configuration control modules built into one system.

2. Adaptive configuration controller with partial hardware reconfiguration: This design incorporates runtime partial hardware reconfiguration technology

3. Adaptive configuration controller with partial hardware reconfiguration working with PID control in software: A gyro sensor is added to the hardware. PID control algorithm is implemented in software. The hardware/software system is used to balance the multirotor on its roll axis. 
The adaptive controller system was tested to find out the variation in power requirement for different multirotor configurations. The design with partial hardware reconfiguration and the model without partial hardware reconfiguration were compared.

In short this thesis demonstrates an innovative multirotor controller with adaptive motor configuration which is implemented using runtime partial hardware reconfiguration technique on Xilinx Zynq System on Chip.

\section{I.5 Summary of Chapters}

Chapter II Materials and Methods: This chapter explains different concepts and technology associated with this project. The key topics explained in the chapter are Partial Hardware Reconfiguration Technology and multirotor basics.

Chapter III Design and Implementation: This chapter explains the overall design process of our adaptive multirotor controller. Different blocks of the design are explained.

Chapter IV Experiments and Results: This chapter presents the experimental data and observations.

Chapter V Conclusion and Future Work: This chapter is about conclusions drawn from the experiments. It also shares recommendations and objectives for future work. 


\section{Chapter II Materials and Methods}

\section{II.1 Overview}

The adaptive multirotor controller is designed with Xilinx Zynq as the target device. Field Programmable Gate Arrays (FPGA) makes hardware prototyping cheaper and faster compared to design and fabrication of Application Specific Integrated Circuit (ASIC) Chips. Xilinx Zynq supports runtime hardware partial reconfiguration. This feature is the key technology driving this project. The multirotor controller uses runtime partial hardware reconfiguration to switch between different multirotor arm configurations. The Zynq prototyping board ZedBoard is used as the base platform for our work. ZedBoard is connected to sensors and actuators through its peripheral modification headers. The flight control board of a commercially available multirotor is replaced with the design developed in this work for testing. A gyro sensor is interfaced with the ZedBoard for implementing a control algorithm in the software side. This section gives a brief description on various technologies and hardware used in this project.

\section{II.2 Xilinx Zynq System on Chip}

Xilinx Zynq System on Chip combines a dual core Cortex-A9 processor with a Xilinx 7 series FPGA fabric [12]. The reasons for choosing Zynq for this work are the increased design speed and support for dynamic partial reconfiguration. The hard processor and associated hard fabricated logic is called processing system (PS) of Zynq. The FPGA fabric is called the programmable logic (PL) (Figure II.1). The processing system of Zynq is hard IP, hence it takes less time to synthesize and implement a design on Zynq compared to other technologies which use soft processors. A soft core processor and associated IP cores has to be synthesized and implemented each time a design prototype needs to be tested. Zynq based development needs only the logic in the PL section to be synthesized making the design process a lot faster. 


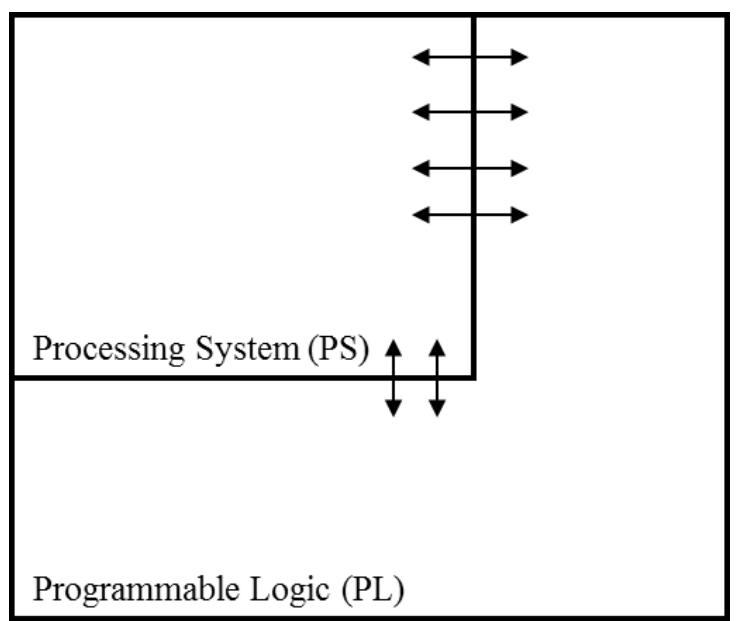

Figure II.1: Zynq Processing System (PS) and Programmable Logic (PL) Zynq System on chip supports a wide range of pre-tested soft intellectual property (IPs) from the Xilinx IP catalog in the Xilinx Embedded Development Kit. The whole process of hardware/software co-design is accelerated by using Zynq SoC. It becomes very easy to implement designs with high level of abstraction. For example, we can design a system to control the speed of a motor which includes both hardware modules to generate speed control signals and software modules to implement control algorithms. The programmable logic of Zynq can effectively implement hardware modules and the processing system can run software modules.

\section{II.3 ZedBoard}

ZedBoard is a popular prototyping board to design and test Zynq based hardware/software systems. ZedBoard houses a XC72020 Zynq device [18]. Xilinx makes the Zynq chip, Avnet is the distributor and Digilent Inc manufactures the board. A wide range of training material on ZedBoard is available online. ZedBoard is the ideal board for somebody new to Zynq based design. Designs for ZedBoard can be developed either using Xilinx ISE Design Suite or the Xilinx Vivado Design Suite. The design or this thesis work was developed using Xilinx ISE design Suite. 


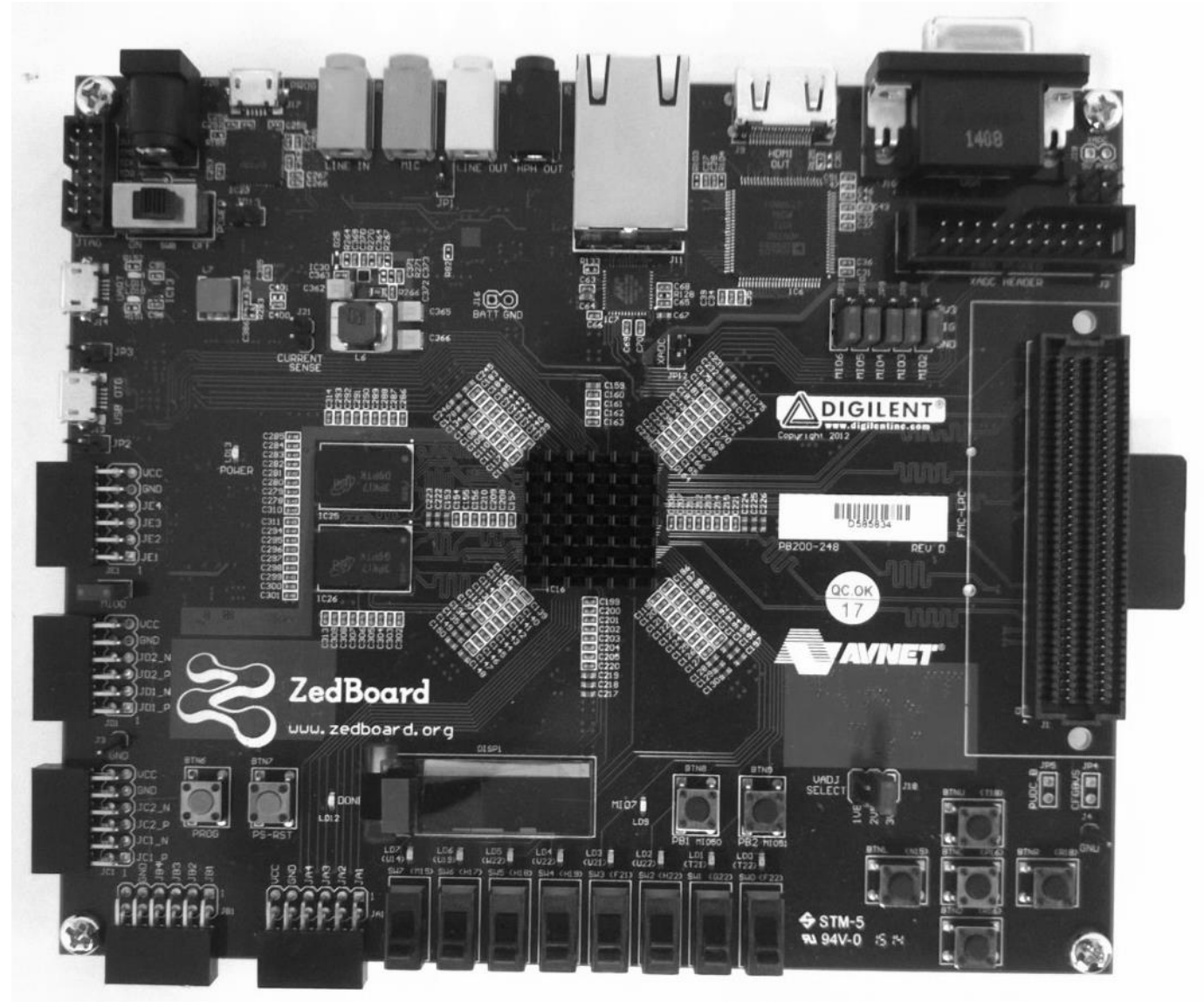

Figure II.2: ZedBoard (Photo taken by author)

ZedBoard is equipped with a variety of peripherals to support a wide range of

hardware/software co-design projects (Figure II.2). The two main hardware features of ZedBoard which are essential for this thesis work are the SD card reader and the Digilent PMOD (Peripheral Modules) compatible headers. ZedBoard kit comes with a 4GB SD card. The SD card can be used either as a non-volatile storage device or as a boot source. There are five PMOD headers on a ZedBoard. Each PMOD header has eight I/O pins, one 3.3V pin, and one Ground pin.

In this thesis project the SD card was used to store the partial bit streams for dynamically reconfiguring the hardware during runtime. The PMOD headers were used to connect a gyro sensor to the design and to connect control signals from the design to external devices. 


\section{II.4 Dynamic Partial Hardware Reconfiguration}

Dynamic partial configuration technology makes it possible to reconfigure parts of a hardware design after it has been implemented on a FPGA fabric. A design which supports dynamic partial hardware reconfiguration will have one or more reconfigurable partitions in the design. Each reconfigurable partition will be associated with one more reconfigurable modules. The rest of the design known as the fixed logic remains unchanged (Figure II.3). The reconfigurable modules need to be created in advance and stored as partial bit streams. The partial bit streams are used to reconfigure a reconfigurable partition as needed. The reconfigurable modules associated with a reconfigurable partition can be used to reconfigure it to change its functional features during runtime [12].

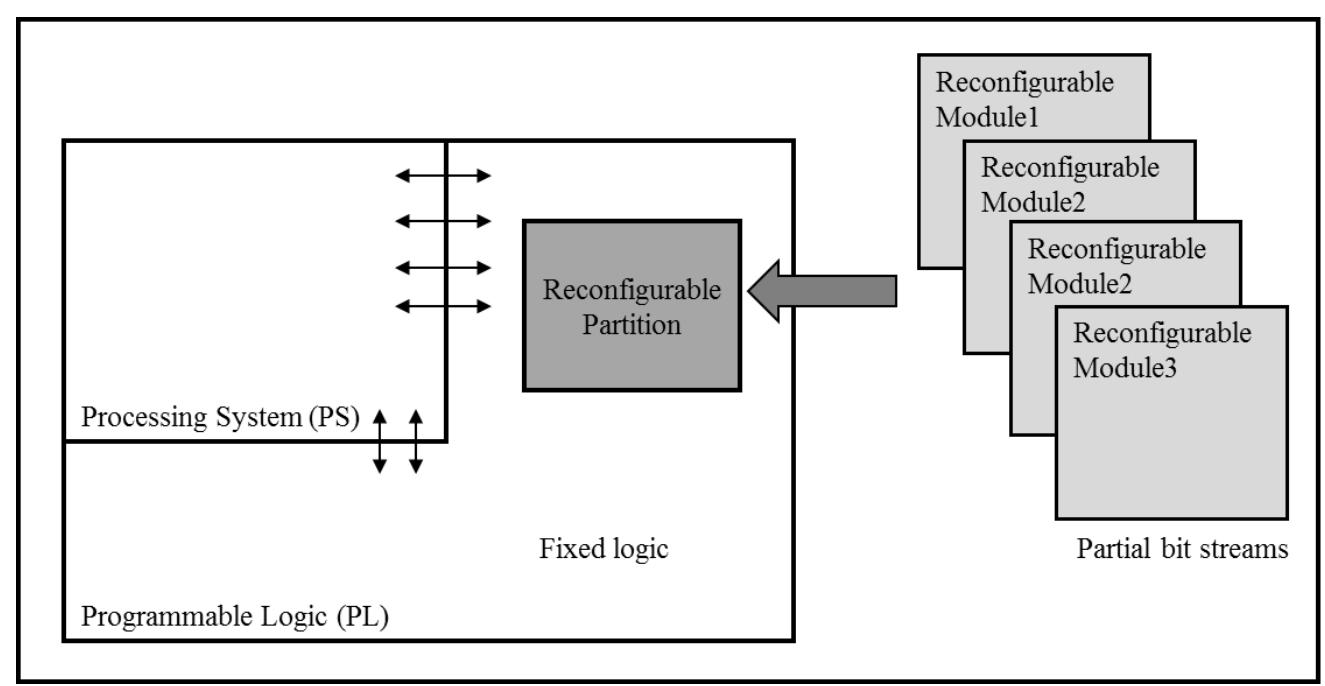

Figure II.3: Dynamic partial hardware reconfiguration

Dynamic partial hardware reconfiguration can reduce size and cost of a design [19]. It also adds the possibility to add new functionalities or features to already existing designs which have a reconfigurable partition [20]. Performance and efficiency of designs can also be improved with the help of dynamic partial reconfiguration technology [21, 22]. Either Xilinx PlanAhead design tool or command line interface has to be used to work with this technology. The synthesis 
of reconfigurable modules has to be done outside PlanAhead. Clock modifying logic has to be avoided in reconfigurable partitions. It should be implemented as a part of the fixed logic. Bidirectional I/O also should be avoided in reconfigurable modules [13].

In this thesis work dynamic partial hardware reconfiguration is used to dynamically switch multirotor arm configurations. A part of the hardware design is implemented as a reconfigurable partition. The hardware logic for each multirotor arm configuration is stored as reconfigurable module partial bit streams. Using dynamic partial reconfiguration we can reduce size of the system compared to a design implemented without runtime partial reconfiguration. In addition it also gives us the flexibility to add additional functionality by adding new reconfigurable modules. Hardware accelerator for software processes can be designed using dynamic partial reconfiguration which makes it possible to modify the accelerator using a partial bit stream instead for recreating the hardware accelerator with the new functionality [23].

\section{II.5 Hardware Components}

\section{II.5.1 Multirotor}

Multirotors have six degrees of freedom (both rotational and translational). However, multirotors are under actuated, which means that they have to turn themselves about their yaw axis to change their horizontal direction of movement. A multirotor can rotate about its center of mass about any of its three axis (Figure II.4). The measures of angle of rotation are called roll, pitch and yaw [16]. Multirotors can be constructed in different motor configurations. Most common multirotor configurations are four motor (Quadcopter), six motor (Hexacopter), and eight motor (Octocopter). It is possible to implement other configurations also. A three motor configuration is possible but it will need a servo mechanism (or additional motorized propeller on the tail motor fixed in the downward direction) on its tail motor to control its yaw. A 
Quadcopter is the simplest configuration, but it will crash if any one of its motors malfunction. A Hexacopter and Octocopter add redundancy to a multirotor making it more reliable. A Hexacopter or an Octocopter can be landed safely even if one of its motors malfunction. Moreover, additional motors add more load carrying capacity to the multirotor. Multirotor technology brings together elements of various domains of engineering.

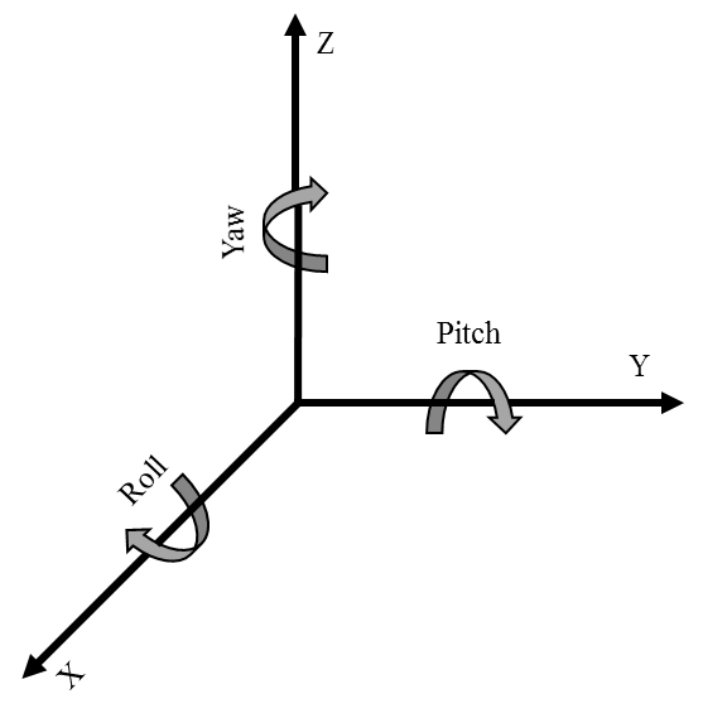

Figure II.4: Roll, pitch, and yaw of a multirotor

The objective of this study is to develop an adaptive controller which can switch between different multirotor arm configurations. Currently, it is not our intention to develop a new multirotor or a multirotor control algorithm. So a commercially available multirotor platform (XAircraft DIY Hexa) was used to test the design.

\section{II.5.2 Gyro Sensor}

A peripheral module (PMOD) gyro sensor from Digilent Inc is used in final design of this work (Figure II.5). The device has a STMicroelectronics L3G4200D MEMS motion sensor. It is a 3 axis gyroscope [24]. It also has a built in temperature sensor. Only the x-axis value is used in the final design as the multirotor is constrained rotate only on its roll axis. 


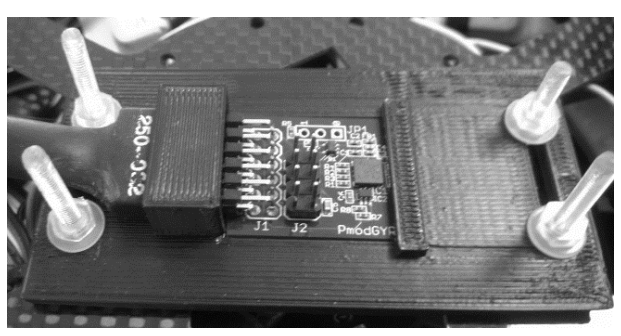

Figure II.5: Digilent PmodGYRO 3-Axis digital gyroscope (Photo taken by author)

\section{II.5.3 Voltage and Current Sensor}

The current sensor Sparkfun AttoPilot Voltage and Current Sense Breakout - 180A is used to take real-time measurement of current and voltage from the system (Figure II.6). The sensor measures current by measuring the voltage drop across a shunt resistor. The measured value is converted to analog voltage value by Texas Instruments INA-169 current shunt monitor [25]. The current sensor is connected in between the battery and the multirotor under test. The sensor is used with an Arduino Nano microcontroller board. Real-time current and voltage measurement is read from a serial monitor.

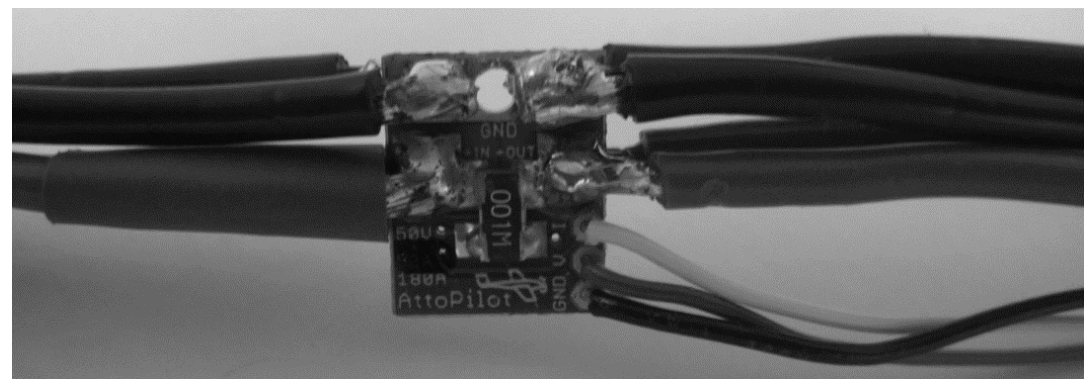

Figure II.6: Sparkfun AttoPilot voltage and current sensor (Photo taken by author) 


\section{Chapter III Design and Implementation}

\section{III.1 Design Overview}

Our design goal was to design a simple hardware/software system to test our hypothesis of the adaptive multirotor controller. First we had to decide which multirotor configurations needed to be included in the scope of this thesis. Then we needed to design the control logic for switching between multirotor configurations. Our base multirotor hardware was a XAircraft DIY HEXA hexacopter. We removed the flight controller board which came with it so that we could connect our controller to the multirotor. The propellers, motors, electronic speed controllers (ESCs), and the power distribution board were the default components. An overview of the design is shown in Figure III.1.

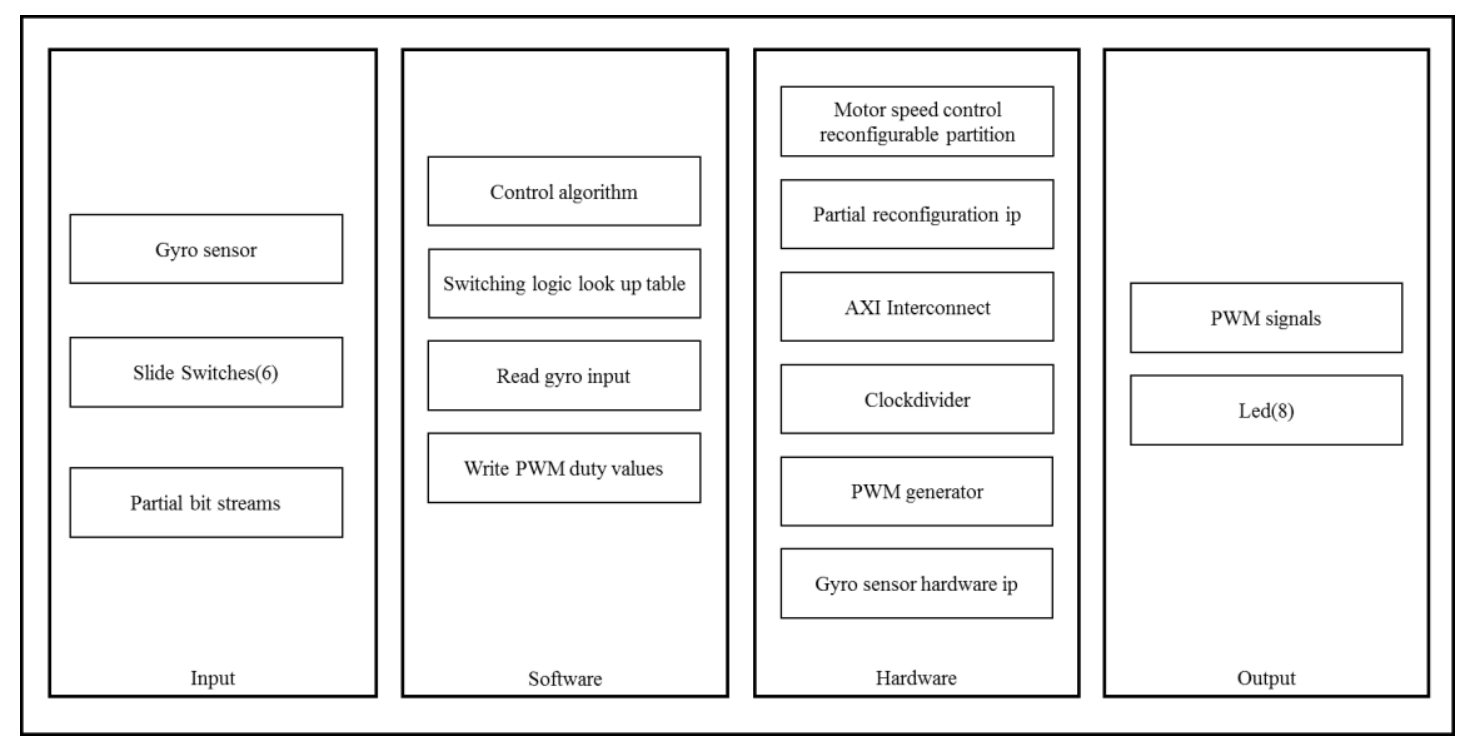

Figure III.1: Design overview

We designed a hardware module which generated pulse width modulated (PWM) signals for the system [26]. We had to make the reconfigurable hardware modules simple as this was our first project in this direction. The partial reconfiguration modules distributed PWM signals to motors depending upon the multirotor configuration implemented by each module. The control algorithm used was proportional integral derivative (PID) and it was implemented on the 
software side. The gyro sensor sent angular velocity data to the processor. The angular velocity data was passed through the PID algorithm to calculate PWM signal duty cycle for the motors.

Six slide switches were used to form the input signal which controls the runtime reconfiguration of the multirotor. The six switches represent six motors (or six multirotor arms). If a switch is turned ON during operation that is interpreted as the motor represented by the switch is available (working/ user wants to use it). Then the multirotor will be configured into a configuration which uses the motor if a stable configuration is possible when the motor is included. If no stable configuration is attainable by including the motor the multirotor stays in the current configuration. If a switch is turned OFF during operation the multirotor is reconfigured into a stable configuration which does not use the motor represented by the switch if such a stable configuration is possible. If no valid configuration is present then all motors in the multirotor are turned off in our current implementation. Further study needs to be done to come up with a better response for this scenario. Section III.3 explains how to differentiate between a valid configuration and an invalid configuration.

The hardware part of the multirotor controller consists of four parts: the processor, the control module with a reconfigurable partition, the gyro sensor IP, and a PWM generator. The software running on the processor interacts with the custom hardware IP module through memory mapped registers. The PWM output generated is distributed to output ports by the control module. One of the peripheral modifications (PMOD) is used as the external pin through which the PWM signals are connected to the electronic speed controllers of the motors. The Digilent Inc Gyro sensor is also connected to one of the PMOD ports. The gyro sensor reading is sent to a memory mapped register and the software reads the data from there. The software writes PWM duty cycle information for the control module in to the registers. The control 
module uses this information to drive the PWM generator to produce PWM signals with the required duty cycle.

\section{III.2 Multirotor Configurations Considered in this Project}

We had to choose the multirotor configurations that could be tested using our experimental setup. Our base system was a hexacopter. Therefore, our choices were a 'six motor' configuration, three 'four motor' configurations, and two 'three motor' configurations. We acknowledge that if we change the configuration starting with six motors the yaw angular momentum will be unbalanced in most of the other configurations. A potential solution for this issue will be to use two motors per arm one pointing upwards and one pointing downwards. It is beyond the scope of this thesis. Further studies need to be done to test this solution.

\section{III.2.1 Six Motor Configuration (1, 2, 3, 4, 5, 6)}

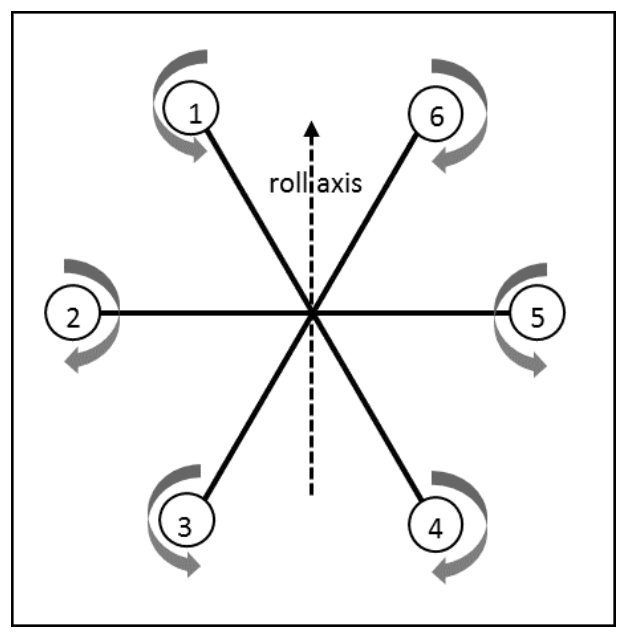

Figure III.2: Six motor configuration

In this configuration all the arms $(1,2,3,4,5,6)$ of the multirotor are used (Figure III.2). PWM signals are sent to all six motors. Our multirotor controller changes the speed of all six motors using PWM signals to keep the multirotor balanced on the roll axis. The angle between each arm of the multirotor is 60 degrees. As the number of motors in each spin direction is equal the yaw angular moments will cancel each other in this configuration. 


\section{III.2.2 Four Motor Configuration $(1,3,4,6)$}

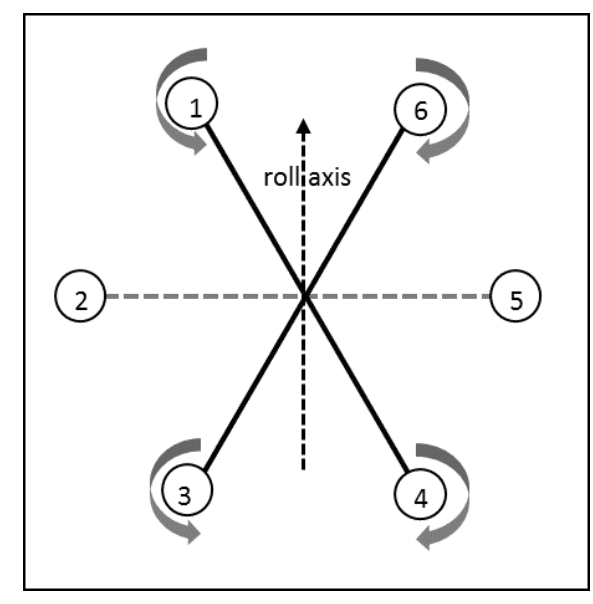

Figure III.3: Four motor configuration $(1,3,4,6)$

In this configuration four out of six arms are used $(1,3,4,6)$ of the multirotor are used (Figure III.3). In this configuration there are two arms on each side of the roll axis. The four arms form a symmetrical configuration with respect to the roll axis. The other two four arm configuration lacks this symmetry with respect to the roll axis (sections III.2.3 and III.2.4). Hence this configuration is the only four arm configuration included in the final design in this study. The other two four arm configurations were used only in the initial reconfiguration experiments.

It must be noted that as our initial multirotor configuration is a hexacopter configuration using six arms, the motor spin directions are set to balance the yaw angular momentum in that configuration. Therefore the yaw momentum may not be zero (when speeds of motors are unequal) when the multirotor is reconfigured into a four arm configuration. In our experimental setup it does not affect our goal of balancing the multirotor in its roll axis as the multirotor is not free to move on its yaw axis. However, in further development using two motors with opposite spin per arm, one facing upward and one facing downward should be a possible solution for this issue. 
The four arms in this configuration form an angle of 30 degrees with the roll axis (Figure III.4). Hence only a part of the thrust from the motors is available for balancing the multirotor along the roll axis. In the case of six motor configuration one motor each on both side (motor 2 and motor 5) supplied their full thrust while the others supplied a part of their thrust for balancing the multirotor on the roll axis.

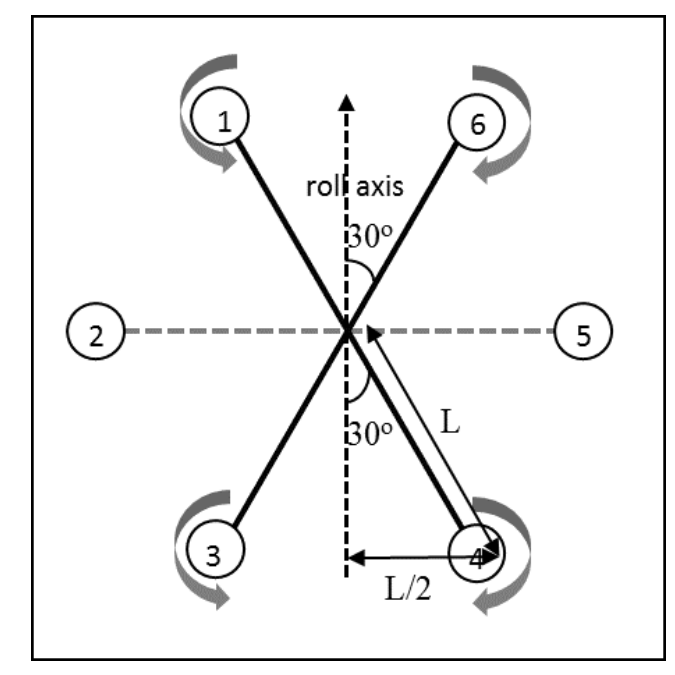

Figure III.4: Torque distribution

Length of arm $=\mathrm{L}$

Thrust of motor $(\mathrm{n})=\mathrm{F}$

Total Torque along the $\operatorname{arm}(\mathrm{n})=\mathrm{L} \times \mathrm{F}=\mathrm{T}_{\text {total }}$

For arms 1, 3, 5, 6 torque parallel to roll axis $=\mathrm{LCos} 30 \times \mathrm{F}=\mathrm{T}_{\text {total }} \times \operatorname{Sqrt}(3) / 2=86 \%$ of $\mathrm{T}_{\text {total }}$ Torque perpendicular to roll axis $=\mathrm{LSin} 30 \times \mathrm{F}=\mathrm{T}_{\text {total }} \times 1 / 2=50 \%$ of $\mathrm{T}_{\text {total }}$

Therefore in this four motor configuration we get $50 \%$ of total torque from each arm to keep the multirotor balanced along the roll axis. 


\section{III.2.3 Four Motor Configurations $(1,2,4,5)$}

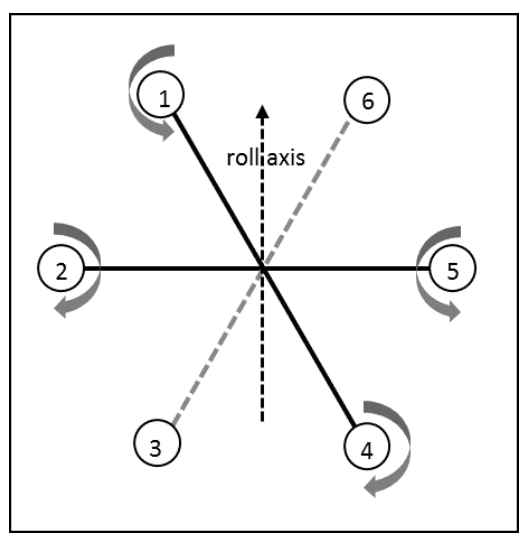

Figure III.5: Four motor configuration $(1,2,4,5)$

In this configuration four out of six arms of the multirotor $(1,2,4,5)$ are used (Figure

III.5). In this configuration the two motors on each side of the roll axis do not form a symmetrical arrangement. This configuration was included in the initial experiments. However, it was not included in the final design of this thesis. This configuration needs to be studied further.

\section{III.2.4 Four Motor Configurations $(2,3,5,6)$}

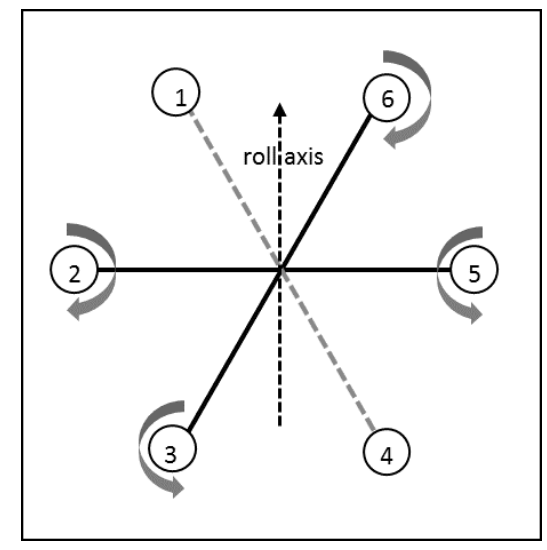

Figure III.6: Four motor configuration 2, 3, 5, 6)

In this configuration four arms $(2,3,5,6)$ are used (Figure III.6). Like the previous 'four motor configuration' this configuration also lacks symmetry in arms placement with respect to the roll axis. This configuration is also not included in our final design. 
It must be also noted that in the last two of the four arm configurations, the orientation of the multirotor with respect to the roll axis has shifted as the active arms are not symmetrically placed. In other words the current roll axis will lose its meaning in the last two configurations. This can be a concern in the future development of this platform. It might be essential for a multirotor to maintain its initial orientation if it is carrying equipment which is calibrated with respect to the initial orientation.

\section{III.2.5 Three Motor Configurations $(1,3,5)$}

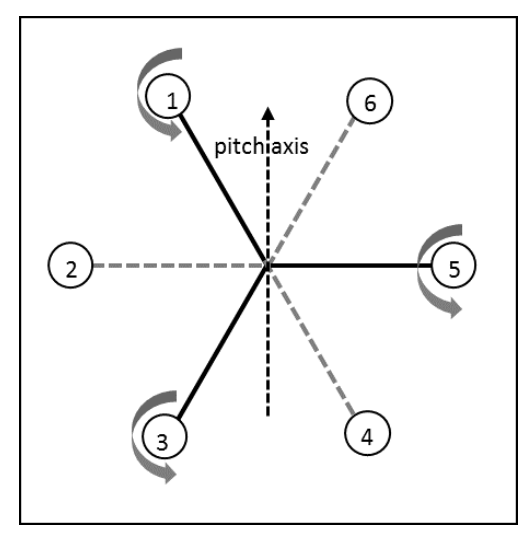

Figure III.7: Three motor configuration $(1,3,5)$

There are two possibilities to form three motor configurations. In one three motor configuration we use the arms 1, 3, and 5 (Figure III.7). In a three motor multirotor configuration we have one active arm on one side and two active arms on the other side. It should be noted that in a conventional three arm multirotor the side that has two active arms is the front. Therefore the axis in our experimental setup should be considered as the 'pitch' axis (instead of the roll axis) of the three arm configuration. The two active arms will provide $50 \%$ of their total thrust each on one side. The single active arm on the other side will provide its full thrust for balancing the multirotor on its pitch axis (the axis of rotation in our experimental setup). This configuration is included in all experiments in this work. 


\section{III.2.6 Three Motor Configurations $(2,4,6)$}

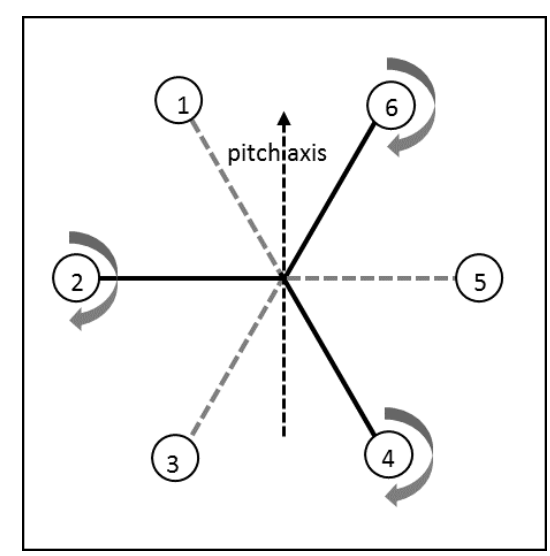

Figure III.8: Three motor configuration $(2,4,6)$

This multirotor configuration uses the arms 2, 4, and 6 (Figure III.8). This configuration has the exact opposite heading of the other three arm multirotor configurations. This configuration also has its pitch axis along the axis of rotation in the experimental setup.

The yaw angular momentum is not balanced in the case of three motor configurations like the four motor configurations. This is because of the same reason that the motor spin directions where chosen for the hexacopter configuration. In one three motor configuration all three motors spin in clockwise direction and in the other all motors spin in the counter clockwise direction. The effect of unbalanced angular momentum is not visible in the current experimental setup as the multirotor is constrained to move about its yaw axis. Using arms with double motors will be a possible solution in future developments. That is each arm will have two motors each, one pointing upwards and one pointing downwards. All motors pointing upwards will spin in one direction and all the motors in pointing downwards will spin in the other direction. In this arrangement the net yaw angular momentum of each arm should be balanced. Hence, if two motors are used per arm, the number of arms in a configuration should not affect the new yaw angular momentum. 


\section{III.3 Control Module Switching Logic}

The control module switching logic works based on the input from a set of slide switches. As we are working with six arm configurations, four arm configurations, and three arm configurations, we use six slide switches (one switch for each motor) on the ZedBoard as our control module selection input. It is assumed that a switch represents whether or not to use an arm in the operation of the multirotor. The on/off state of the switches has to be interpreted as a six input set so that the software can decide the best multirotor configuration possible with the available arms.

As there are six arms represented by six switches there are $64\left(2^{6}\right)$ possible states for the six bit switch input. That is the switch input signal can take any value between from ' 000000 ' (all switches off) to ' 111111 ' (all switches on). When all the switches are on the input read by the software will be ' 111111 ' and the best possible multirotor configuration is the six arm (hexacopter) configuration. An input of all ones will trigger the system to load the reconfigurable partition into the custom hardware with the partial bit stream associated with six arm configuration. If the input is a value for which a valid multirotor configuration is available the respective partial bit stream will be loaded into the reconfigurable hardware partition.

The software differentiates between an input which form a valid configuration and an input which cannot form a valid flight configuration using a look up table. The lookup table stores all possible input values (6 bit values from switches) and the respective output configurations ( 6 bit). The six bits in the output indicate which arms are to be used in the configuration if a valid configuration is available ( 1 use the arm, 0 do not use the arm). 


\begin{tabular}{|c|c|c|c|c|c|c|c|c|c|c|c|c|c|}
\hline \multicolumn{14}{|c|}{ Multicopter Adaptive Flight using Partial Hardware Reconfiguration: Reconfiguration Logic } \\
\hline SW6 & SW5 & SW4 & SW3 & SW2 & SW1 & Decimal Code & M6 & M5 & M4 & M3 & $\mathrm{M} 2$ & M1 & Decimal Code \\
\hline 0 & 0 & 0 & 0 & 0 & 0 & 0 & 0 & 0 & 0 & 0 & 0 & 0 & 0 \\
\hline 0 & 0 & 0 & 0 & 0 & 1 & 1 & 0 & 0 & 0 & 0 & 0 & 0 & 0 \\
\hline 0 & 0 & 0 & 0 & 1 & 0 & 2 & 0 & 0 & 0 & 0 & 0 & 0 & 0 \\
\hline 0 & 0 & 0 & 0 & 1 & 1 & 3 & 0 & 0 & 0 & 0 & 0 & 0 & 0 \\
\hline 0 & 0 & 0 & 1 & 0 & 0 & 4 & 0 & 0 & 0 & 0 & 0 & 0 & 0 \\
\hline 0 & 0 & 0 & 1 & 0 & 1 & 5 & 0 & 0 & 0 & 0 & 0 & 0 & 0 \\
\hline 0 & 0 & 0 & 1 & 1 & 0 & 6 & 0 & 0 & 0 & 0 & 0 & 0 & 0 \\
\hline 0 & 0 & 0 & 1 & 1 & 1 & 7 & 0 & 0 & 0 & 0 & 0 & 0 & 0 \\
\hline 0 & 0 & 1 & 0 & 0 & 0 & 8 & 0 & 0 & 0 & 0 & 0 & 0 & 0 \\
\hline 0 & 0 & 1 & 0 & 0 & 1 & 9 & 0 & 0 & 0 & 0 & 0 & 0 & 0 \\
\hline 0 & 0 & 1 & 0 & 1 & 0 & 10 & 0 & 0 & 0 & 0 & 0 & 0 & 0 \\
\hline 0 & 0 & 1 & 0 & 1 & 1 & 11 & 0 & 0 & 0 & 0 & 0 & 0 & 0 \\
\hline 0 & 0 & 1 & 1 & 0 & 0 & 12 & 0 & 0 & 0 & 0 & 0 & 0 & 0 \\
\hline 0 & 0 & 1 & 1 & 0 & 1 & 13 & 0 & 0 & 0 & 0 & 0 & 0 & 0 \\
\hline 0 & 0 & 1 & 1 & 1 & 0 & 14 & 0 & 0 & 0 & 0 & 0 & 0 & 0 \\
\hline 0 & 0 & 1 & 1 & 1 & 1 & 15 & 0 & 0 & 0 & 0 & 0 & 0 & 0 \\
\hline 0 & 1 & 0 & 0 & 0 & 0 & 16 & 0 & 0 & 0 & 0 & 0 & 0 & 0 \\
\hline 0 & 1 & 0 & 0 & 0 & 1 & 17 & 0 & 0 & 0 & 0 & 0 & 0 & 0 \\
\hline 0 & 1 & 0 & 0 & 1 & 0 & 18 & 0 & 0 & 0 & 0 & 0 & 0 & 0 \\
\hline 0 & 1 & 0 & 0 & 1 & 1 & 19 & 0 & 0 & 0 & 0 & 0 & 0 & 0 \\
\hline 0 & 1 & 0 & 1 & 0 & 0 & 20 & 0 & 0 & 0 & 0 & 0 & 0 & 0 \\
\hline 0 & 1 & 0 & 1 & 0 & 1 & 21 & 0 & 1 & 0 & 1 & 0 & 1 & 21 \\
\hline 0 & 1 & 0 & 1 & 1 & 0 & 22 & 0 & 0 & 0 & 0 & 0 & 0 & 0 \\
\hline 0 & 1 & 0 & 1 & 1 & 1 & 23 & 0 & 1 & 0 & 1 & 0 & 1 & 21 \\
\hline 0 & 1 & 1 & 0 & 0 & 0 & 24 & 0 & 0 & 0 & 0 & 0 & 0 & 0 \\
\hline 0 & 1 & 1 & 0 & 0 & 1 & 25 & 0 & 0 & 0 & 0 & 0 & 0 & 0 \\
\hline 0 & 1 & 1 & 0 & 1 & 0 & 26 & 0 & 0 & 0 & 0 & 0 & 0 & 0 \\
\hline 0 & 1 & 1 & 0 & 1 & 1 & 27 & 0 & 1 & 1 & 0 & 1 & 1 & 27 \\
\hline 0 & 1 & 1 & 1 & 0 & 0 & 28 & 0 & 0 & 0 & 0 & 0 & 0 & 0 \\
\hline 0 & 1 & 1 & 1 & 0 & 1 & 29 & 0 & 1 & 0 & 1 & 0 & 1 & 21 \\
\hline 0 & 1 & 1 & 1 & 1 & 0 & 30 & 0 & 0 & 0 & 0 & 0 & 0 & 0 \\
\hline 0 & 1 & 1 & 1 & 1 & 1 & 31 & 0 & 1 & 1 & 0 & 1 & 1 & 27 \\
\hline 1 & 0 & 0 & 0 & 0 & 0 & 32 & 0 & 0 & 0 & 0 & 0 & 0 & 0 \\
\hline 1 & 0 & 0 & 0 & 0 & 1 & 33 & 0 & 0 & 0 & 0 & 0 & 0 & 0 \\
\hline 1 & 0 & 0 & 0 & 1 & 0 & 34 & 0 & 0 & 0 & 0 & 0 & 0 & 0 \\
\hline 1 & 0 & 0 & 0 & 1 & 1 & 35 & 0 & 0 & 0 & 0 & 0 & 0 & 0 \\
\hline 1 & 0 & 0 & 1 & 0 & 0 & 36 & 0 & 0 & 0 & 0 & 0 & 0 & 0 \\
\hline 1 & 0 & 0 & 1 & 0 & 1 & 37 & 0 & 0 & 0 & 0 & 0 & 0 & 0 \\
\hline 1 & 0 & 0 & 1 & 1 & 0 & 38 & 0 & 0 & 0 & 0 & 0 & 0 & 0 \\
\hline 1 & 0 & 0 & 1 & 1 & 1 & 39 & 0 & 0 & 0 & 0 & 0 & 0 & 0 \\
\hline 1 & 0 & 1 & 0 & 0 & 0 & 40 & 0 & 0 & 0 & 0 & 0 & 0 & 0 \\
\hline 1 & 0 & 1 & 0 & 0 & 1 & 41 & 0 & 0 & 0 & 0 & 0 & 0 & 0 \\
\hline 1 & 0 & 1 & 0 & 1 & 0 & 42 & 1 & 0 & 1 & 0 & 1 & 0 & 42 \\
\hline 1 & 0 & 1 & 0 & 1 & 1 & 43 & 1 & 0 & 1 & 0 & 1 & 0 & 42 \\
\hline 1 & 0 & 1 & 1 & 0 & 0 & 44 & 0 & 0 & 0 & 0 & 0 & 0 & 0 \\
\hline 1 & 0 & 1 & 1 & 0 & 1 & 45 & 1 & 0 & 1 & 1 & 0 & 1 & 45 \\
\hline 1 & 0 & 1 & 1 & 1 & 0 & 46 & 1 & 0 & 1 & 0 & 1 & 0 & 42 \\
\hline 1 & 0 & 1 & 1 & 1 & 1 & 47 & 1 & 0 & 1 & 1 & 0 & 1 & 45 \\
\hline 1 & 1 & 0 & 0 & 0 & 0 & 48 & 0 & 0 & 0 & 0 & 0 & 0 & 0 \\
\hline 1 & 1 & 0 & 0 & 0 & 1 & 49 & 0 & 0 & 0 & 0 & 0 & 0 & 0 \\
\hline 1 & 1 & 0 & 0 & 1 & 0 & 50 & 0 & 0 & 0 & 0 & 0 & 0 & 0 \\
\hline 1 & 1 & 0 & 0 & 1 & 1 & 51 & 0 & 0 & 0 & 0 & 0 & 0 & 0 \\
\hline 1 & 1 & 0 & 1 & 0 & 0 & 52 & 0 & 0 & 0 & 0 & 0 & 0 & 0 \\
\hline 1 & 1 & 0 & 1 & 0 & 1 & 53 & 0 & 1 & 0 & 1 & 0 & 1 & 21 \\
\hline 1 & 1 & 0 & 1 & 1 & 0 & 54 & 1 & 1 & 0 & 1 & 1 & 0 & 54 \\
\hline 1 & 1 & 0 & 1 & 1 & 1 & 55 & 1 & 1 & 0 & 1 & 1 & 0 & 54 \\
\hline 1 & 1 & 1 & 0 & 0 & 0 & 56 & 0 & 0 & 0 & 0 & 0 & 0 & 0 \\
\hline 1 & 1 & 1 & 0 & 0 & 1 & 57 & 0 & 0 & 0 & 0 & 0 & 0 & 0 \\
\hline 1 & 1 & 1 & 0 & 1 & 0 & 58 & 1 & 0 & 1 & 0 & 1 & 0 & 42 \\
\hline 1 & 1 & 1 & 0 & 1 & 1 & 59 & 0 & 1 & 1 & 0 & 1 & 1 & 27 \\
\hline 1 & 1 & 1 & 1 & 0 & 0 & 60 & 0 & 0 & 0 & 0 & 0 & 0 & 0 \\
\hline 1 & 1 & 1 & 1 & 0 & 1 & 61 & 1 & 0 & 1 & 1 & 0 & 1 & 45 \\
\hline 1 & 1 & 1 & 1 & 1 & 0 & 62 & 1 & 1 & 0 & 1 & 1 & 0 & 54 \\
\hline 1 & 1 & 1 & 1 & 1 & 1 & 63 & 1 & 1 & 1 & 1 & 1 & 1 & 63 \\
\hline
\end{tabular}

Table III.1: Multirotor control switching logic input and output configurations 
Table III.1 lists all possible input from the switches. All the rows which are highlighted in gray do not have a stable (valid) configuration. In that case the multirotor controller will be reconfigured with a control module which supplies a low PWM duty cycle to the motors such that no motors will spin. The remaining 18 configurations are the inputs which can get a valid configuration. For example, the input ' 101111 ' (decimal code 47) means that the fifth arm should not be used (or not working). It is up to the system to check if there are any valid configurations available without using the arm for which the input bit is ' 0 '. A valid configuration can be achieved by turning of the diametrically opposite arm (arm 2). That logic is represented as the respective output '101101' (decimal code 45) in the output column.

The result of an input combination is said to be in an unsteady (invalid) state, if a balanced arm configuration cannot be formed using the available arms (Figure III.9).

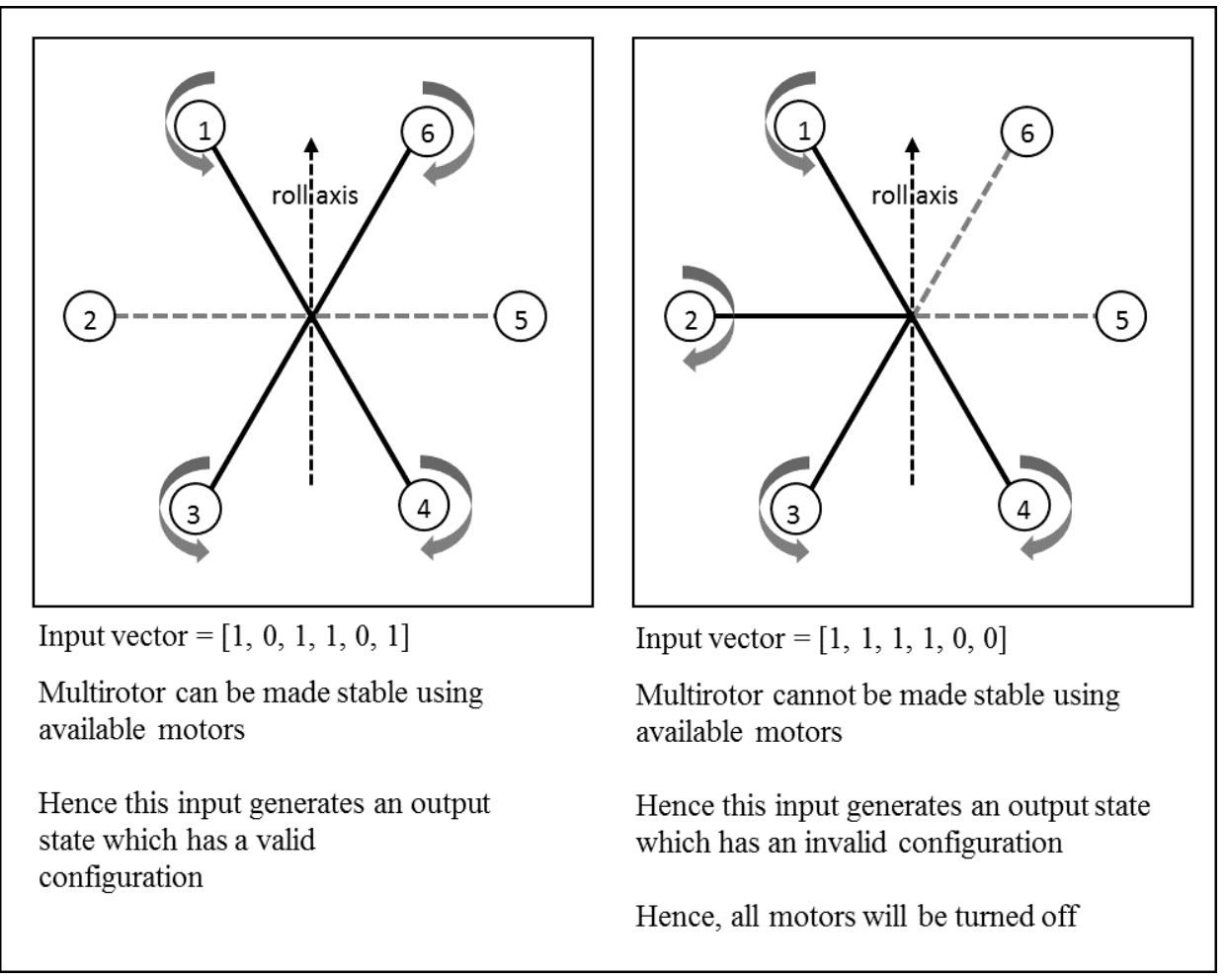

Figure III.9: A valid configuration (left), an invalid configuration (right) 
There are 18 input combinations for which a steady multirotor configuration exists. As discussed in the section III.3 there are one 'six arm' configuration, three 'four arm' configurations, and two 'three arm' configuration. The 18 input combinations are mapped to one of the six valid multirotor configurations.

\begin{tabular}{|c|c|c|c|c|c|c|c|c|c|c|c|c|c|c|c|}
\hline \multicolumn{10}{|c|}{ Multicopter Adaptive Flight using Partial Hardware Reconfiguration: Reconfiguration Logic } \\
\hline SW6 & SW5 & SW4 & SW3 & SW2 & SW1 & Decimal Code & M6 & M5 & M4 & M3 & M2 & M1 & Decimal Code & Multirotor Configuration \\
\hline 0 & 1 & 0 & 1 & 0 & 1 & 21 & 0 & 1 & 0 & 1 & 0 & 1 & 21 & Three Arm $(1,3,5)$ \\
\hline 0 & 1 & 0 & 1 & 1 & 1 & 23 & 0 & 1 & 0 & 1 & 0 & 1 & 21 & Three Arm $(1,3,5)$ \\
\hline 0 & 1 & 1 & 0 & 1 & 1 & 27 & 0 & 1 & 1 & 0 & 1 & 1 & 27 & Four Arm $(1,2,4,5)$ \\
\hline 0 & 1 & 1 & 1 & 0 & 1 & 29 & 0 & 1 & 0 & 1 & 0 & 1 & 21 & Three Arm $(1,3,5)$ \\
\hline 0 & 1 & 1 & 1 & 1 & 1 & 31 & 0 & 1 & 1 & 0 & 1 & 1 & 27 & Four Arm $(1,2,4,5)$ \\
\hline 1 & 0 & 1 & 0 & 1 & 0 & 42 & 1 & 0 & 1 & 0 & 1 & 0 & 42 & Three Arm $(2,4,6)$ \\
\hline 1 & 0 & 1 & 0 & 1 & 1 & 43 & 1 & 0 & 1 & 0 & 1 & 0 & 42 & Three Arm $(2,4,6)$ \\
\hline 1 & 0 & 1 & 1 & 0 & 1 & 45 & 1 & 0 & 1 & 1 & 0 & 1 & 45 & Four Arm $(1,3,4,6)$ \\
\hline 1 & 0 & 1 & 1 & 1 & 0 & 46 & 1 & 0 & 1 & 0 & 1 & 0 & 42 & Three Arm $(2,4,6)$ \\
\hline 1 & 0 & 1 & 1 & 1 & 1 & 47 & 1 & 0 & 1 & 1 & 0 & 1 & 45 & Four Arm $(1,3,4,6)$ \\
\hline 1 & 1 & 0 & 1 & 0 & 1 & 53 & 0 & 1 & 0 & 1 & 0 & 1 & 21 & Three Arm $(1,3,5)$ \\
\hline 1 & 1 & 0 & 1 & 1 & 0 & 54 & 1 & 1 & 0 & 1 & 1 & 0 & 54 & Four Arm $(2,3,5,6)$ \\
\hline 1 & 1 & 0 & 1 & 1 & 1 & 55 & 1 & 1 & 0 & 1 & 1 & 0 & 54 & Four Arm $(2,3,5,6)$ \\
\hline 1 & 1 & 1 & 0 & 1 & 0 & 58 & 1 & 0 & 1 & 0 & 1 & 0 & 42 & Three Arm $(2,4,6)$ \\
\hline 1 & 1 & 1 & 0 & 1 & 1 & 59 & 0 & 1 & 1 & 0 & 1 & 1 & 27 & Four Arm $(1,2,4,5)$ \\
\hline 1 & 1 & 1 & 1 & 0 & 1 & 61 & 1 & 0 & 1 & 1 & 0 & 1 & 45 & Four Arm $(1,3,4,6)$ \\
\hline 1 & 1 & 1 & 1 & 1 & 0 & 62 & 1 & 1 & 0 & 1 & 1 & 0 & 54 & Four Arm $(2,3,5,6)$ \\
\hline 1 & 1 & 1 & 1 & 1 & 1 & 63 & 1 & 1 & 1 & 1 & 1 & 1 & 63 & Six Arm $(1,2,3,4,5,6)$ \\
\hline all other input combinations & $\mathrm{X}$ & 0 & 0 & 0 & 0 & 0 & 0 & 0 & no configuration \\
\hline
\end{tabular}

Table III.2: Mapping of input switch values to a multirotor arm configuration

Table III.2 is used to setup a look up table in the software to facilitate the multirotor configuration switching. The decimal code of the output combinations are stored in an array. For ease of access the array has 64 indexed locations. Each location will store the output decimal code if that input combination has a valid multirotor configuration. The input (switches) value is considered as a 6 bit binary number. The decimal equivalent of the input combination is used as the index for the array. The value stored in the array index is looked up. Then based upon the decimal code obtained from the array the control module in the hardware is reconfigured with the corresponding partial bit stream. All input combinations other than the 18 valid ones stores 
the value ' 0 ', indicating that those combination cannot have a stable multirotor configuration. In that case the reconfigurable partition in the controller is configured with a module which will turn all the motors off.

\section{III.4 Hardware Design}

\section{III.4.1 Design0: Simple Design without Partial Hardware Reconfiguration}

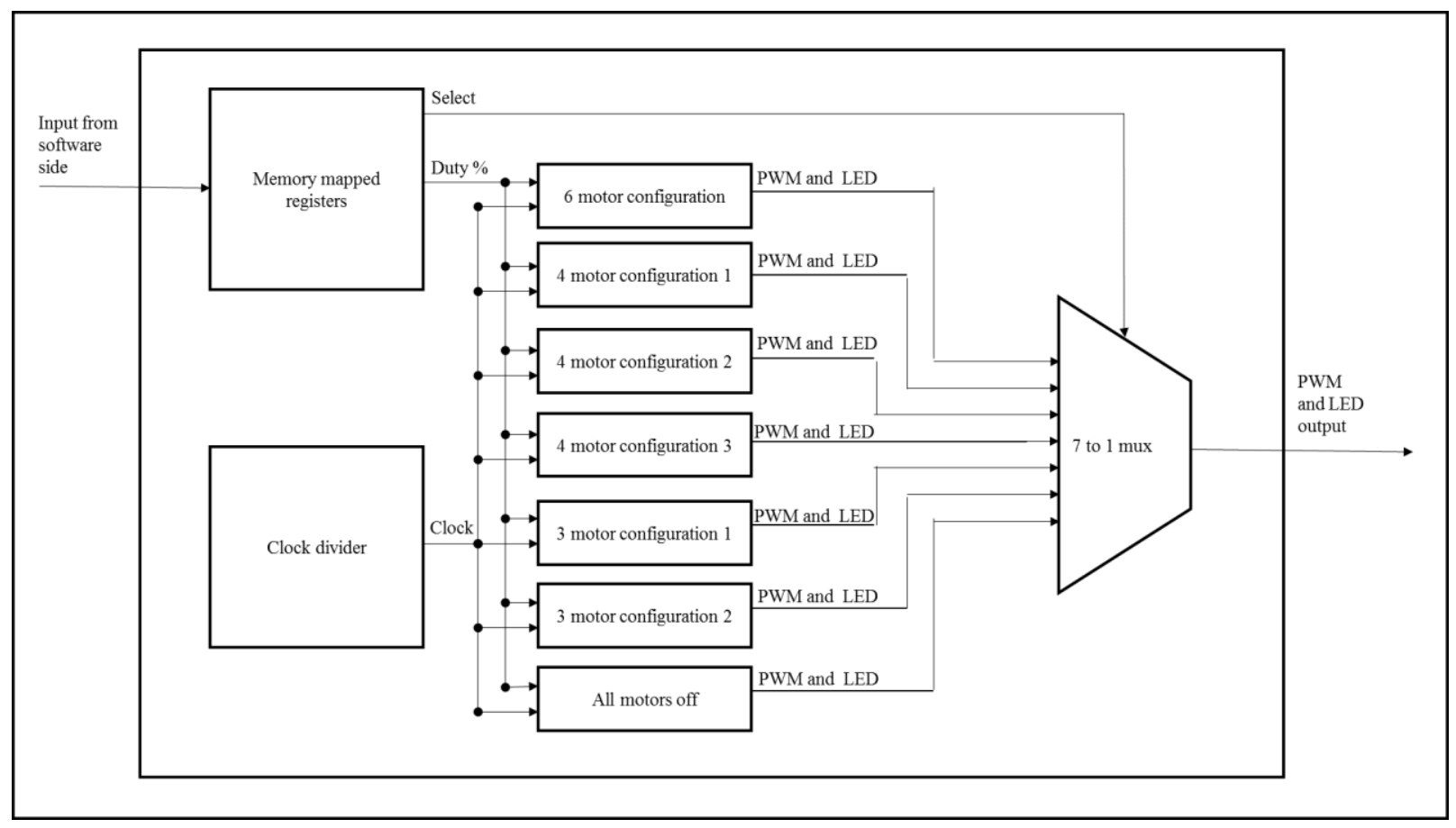

Figure III.10: Hardware design without partial reconfiguration. Flat design: all seven configurations are connected in through a 7-to-1 multiplexer to the output

This hardware was designed to act as a base design for this project. The main goal is to compare the FPGA resources utilization of controller designs with and without partial hardware reconfiguration. In this design all seven multirotor configurations (six valid and one all motor off configurations) exist concurrently in the FPGA fabric (Figure III.10). The top module with a 7to- 1 multiplexer is used to route the input and output signals the control module of choice. This design did not have the gyro sensor IP. Hence, no tests were done on this design with PID algorithm running in software. 
This design communicated with the software side through memory mapped resisters. In this design the PWM duty cycle can be set from the software and the PWM generator produced the PWM signals. Then the PWM signals were routed to the external PMOD pins through the multiplexed control modules.

\section{III.4.2 Design1: Hardware Design with a Reconfigurable Partition}

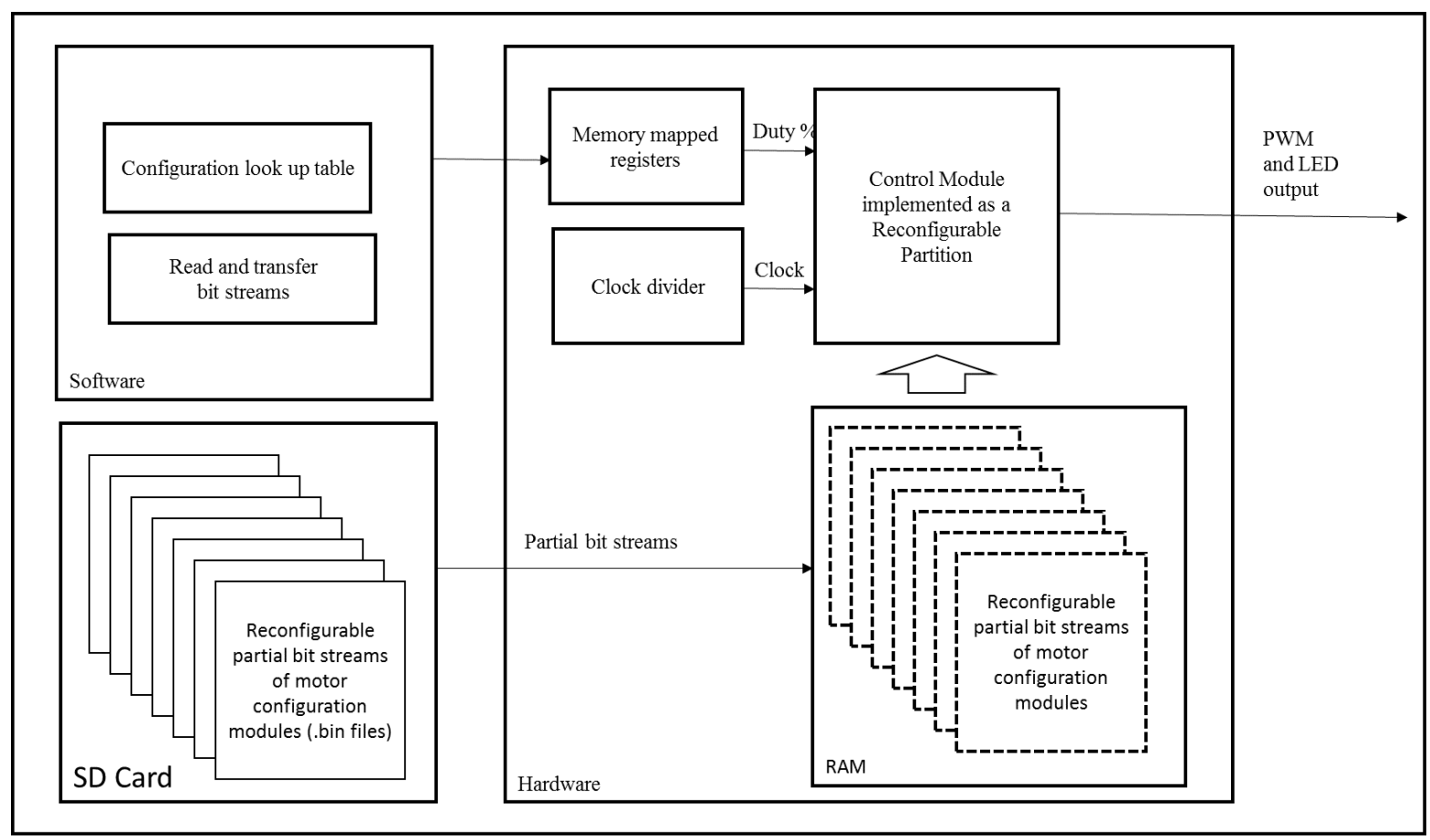

Figure III.11: Hardware design with a reconfigurable partition, seven different configuration are implemented as partial bit streams

In this hardware design we introduce runtime partial hardware reconfiguration. The custom multirotor controller hardware IP have a reconfigurable partition which is configured with one of the partial bit streams (Figure III.11). This design uses less FPGA real estate as only one of the control modules exists in the FPGA fabric at any given time.

This design does not use the gyro sensor. Hence, the software side does not implement the PID algorithm in this design also. The objective of this design is to compare the FPGA resource utilization of the designs with and without runtime partial hardware reconfiguration. 
The seven control configurations are compiled into partial bit streams and stored in a SD card. The partial bit streams are stored as '.bin' files in the SD card. In this design the software reads the partial bit streams stored in the SD card into the RAM. The bit streams are passed to the reconfigurable partition using the processor configuration access port (PCAP) hardware IP of Zynq.

\section{III.4.3 Design2: Prototype with PID and Gyro sensor}

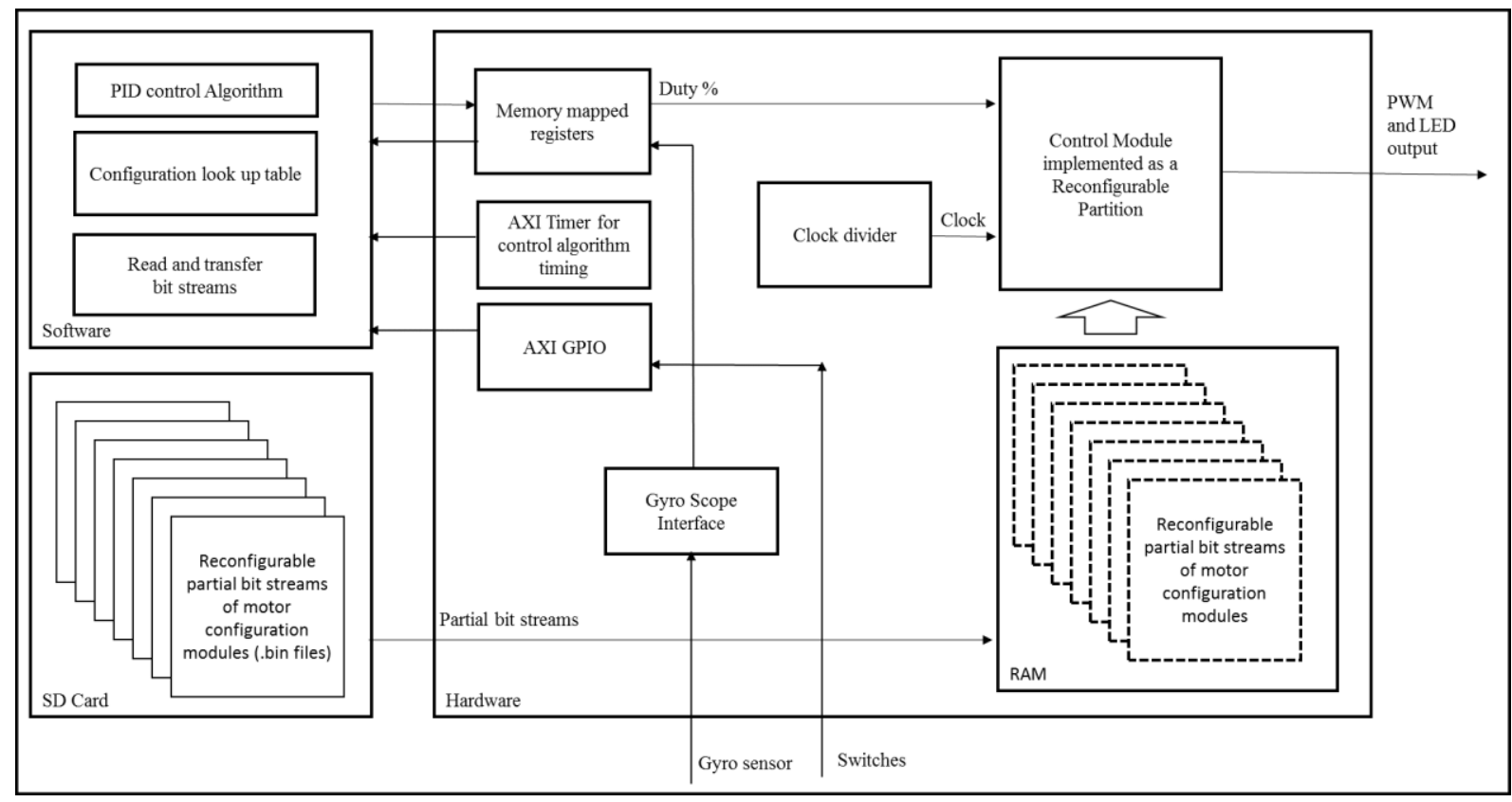

Figure III.12: Gyro sensor and timer added to hardware design which already has a reconfigurable partition

This design is an updated version of the previous design (Figure III.12). In this iteration the design reads angular velocity of the multirotor in all three axis. PID control algorithm is implemented in software to process the angular velocity of the multirotor about the roll axis. Like the previous version this design uses the runtime partial reconfiguration feature of the Zynq chip to reconfigure multirotor control modules. The PID parameters were tuned without making a mathematical model for the system. Therefore the PID parameters may not be the ideal values for the system. 
In this work as the multirotor can move only on its roll axis, only the angular velocity along the $\mathrm{x}$ axis is relevant. The angular velocity is processed using the PID control algorithm to balance the multirotor on its roll axis. The axis of rotation is the roll axis for the one six motor configuration and for the three four motor configurations. However, technically the axis of rotation becomes the pitch axis for the two three motor configurations in our experimental setup. Currently the base throttle (specified as PWM duty cycle) value to the motors is set as a constant. The range of duty values for the PWM signal are 5\% and 11\%. These values are based on the maximum and minimum PWM signals produced by the throttle stick of DX7Se radio transmitter. The multirotor is fed the initial throttle PWM duty cycle at first. Then PID algorithm is used to calculate error correction values. The PID function uses the input from the gyro sensor as the error. The objective of the PID algorithm is to minimize the error registered by the gyro sensor. When the multirotor is balanced, ideally the gyro sensor should output 0 . However, due to vibration of the multirotor the gyro sensor constantly sends a reading and it almost never sends out zero. The PID term, the output of the PID function is used to modify the duty cycle for motors on each side of the roll axis.

Two of the four arm configurations were not used in this design. The four arm configuration $(1,3,4$, and 6$)$ was the only one used in this design. In the other two configurations none of their axis of orientation (yaw, pitch, and roll) align with the axis of the experimental setup. Hence, it is technically meaningless to try to balance those two configuration about an axis which is at an angle to the arm configuration. Therefore this hardware design incorporated five multirotor reconfiguration modules. One Six arm configuration, one four arm configuration, two three arm configurations and a configuration which turned off all motors. 
As this design uses runtime partial hardware reconfiguration like the previous version of the hardware, the clock divider was set up as a part of the static hardware logic. However, the PWM generator module was a part of the reconfigurable module. This is because that the number of instances of the PWM modules changes depending upon the type of multirotor configuration being implemented. In addition the gyro sensor hardware IP was also a part of the static hardware logic.

\section{III.4.4 Design of Individual Control Modules}

There is a control module design associated with each multirotor configuration (Figure III.13). These control modules are used to configure the reconfigurable partition in Design 1 and Design 2. In Design 0 the individual control modules were implemented as concurrent logic on the FPGA. The functions of the control modules are:

1. Generate PWM signals with respect to PWM duty values set by the software

2. Distribute PWM signals properly to active motors in the configuration

3. Generate output for LEDs to indicate the active motors in the current configuration The control modules have PWM generators as sub modules. As we are interested in the motion of our multirotor about its roll axis, we did not setup separate PWM generators for each motor. All the left side motors are controlled by one PWM generator and all the right side motors are connected to another PWM generator. The 'six motor' configuration has two PWM generators. One for generating signals for left side motors and the other for right side motors. For all the other configurations there are three PWM generator sub modules. One for the active motors on the left side, one for active motors on the right side, and one for generating a $5 \%$ PWM signal for inactive motors in the current configuration. A PWM signal of 5\% will keep the motors at rest. Each control module outputs the six PWM signals as a six bit vector. 


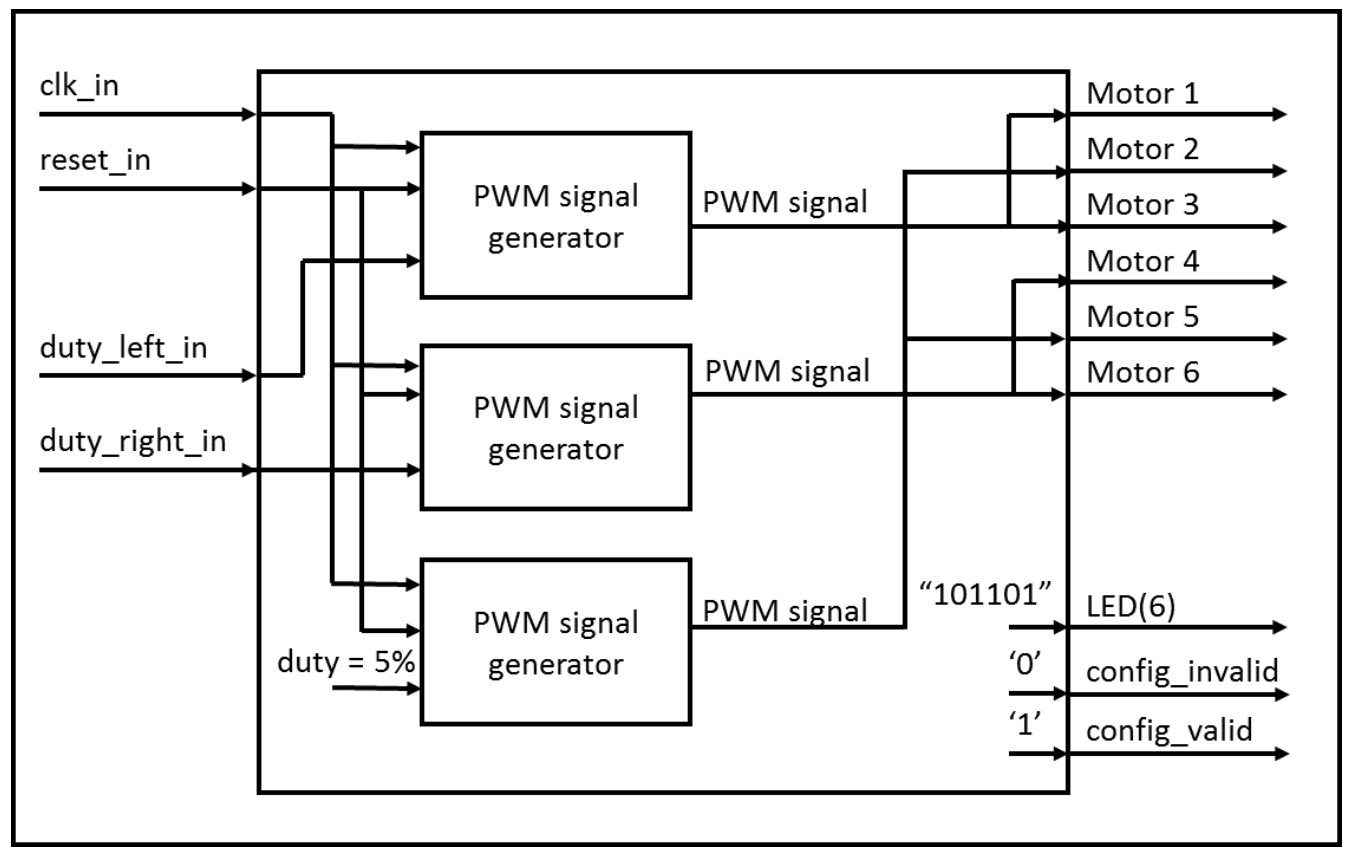

Figure III.13: Structure of a reconfigurable control module 'four motor 1'(1, 3, 4, 6)

Each control module also outputs an eight bit vector to the LEDs. The first six bit indicate which all of the six motors are active in the current configuration. The seventh bit will be ' 1 ' (and all the other bits ' 0 ') for an invalid configuration. The eighth bit is set to ' 1 ' for all valid configurations.

\section{III.4.5 Design of PWM Generator}

The motors of the multirotor are driven using Pulse Width Modulated (PWM) signals [26]. The design parameters of the PWM generator (Figure III.14) were set by measuring the PWM signals produced by the radio controller (DX7Se) using an oscilloscope. The frequency of the PWM signals generated by the radio controller was $45 \mathrm{~Hz}$. The DX7Se radio controller produced PWM signals with a duty of $11 \%$ when the throttle stick was at the maximum position. The duty was $5 \%$ when the stick was at the min position. The duty parameter of a PWM signal determines the strength of a PWM signal. A 100\% PWM signal will be a signal which is high throughout each cycle. 
The hardware under development used was using a base clock of $100 \mathrm{MHz}$. Hence clock dividers were used to scale down the source clock depending upon the period of the PWM signal to be generated. The frequency of the output PWM signal was chosen as $50 \mathrm{~Hz}$ as it was a value close to $45 \mathrm{~Hz}$ which could be easily generated from a scaled down clock derived from the base clock of $100 \mathrm{MHz}$.

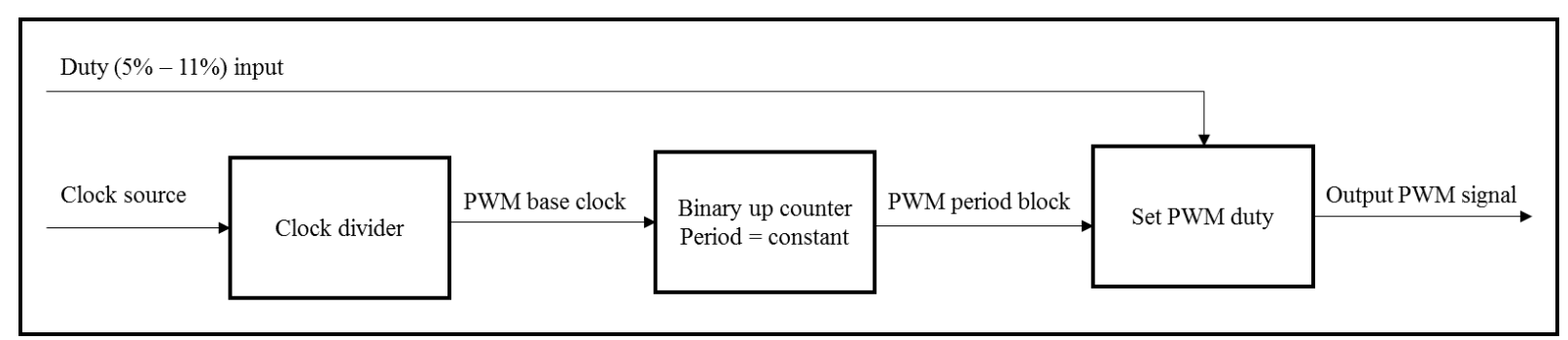

Figure III.14: PWM generator design

The period parameter of the PWM generator was set depending upon the resolution of PWM signal needed. As the minimum and maximum duty values are $5 \%$ and $11 \%$, if the period is 100 , there will be only seven discrete PWM values which can be generated using the controller. It is almost impossible to design a controller using such a low resolution. Moreover the PWM generator module does not work with floating point values. Therefore, in order to get better resolution in PWM duty values without changing the PWM output frequency we increased the period parameter of the PWM generator. Increasing the period by an order of 10 (period = 1000) the maximum duty becomes 110 (11\% of 1000). The minimum duty at this period will be 50. This will give us 70 PWM duty levels $(5.0 \%, 5.1 \% \ldots 11 \%)$.

In our final design we set the period to 50,000,000. This will give us a minimum duty cycle of 50,000 (5\%) and a maximum duty cycle of 110,000 (11\%) (Table III.3). We were tuning the PID algorithm for balancing the multirotor without mathematically modelling the system. In addition we were not passing any floating point values to hardware side. Therefore we had to 
have enough resolution in our control signal so that we could tune the controller by making small adjustments.

\begin{tabular}{|l|l|l|l|l|l|}
\hline source clk (Hz) & PWM base clk (Hz) & PWM output frequency $(\mathrm{Hz})$ & PWM period $(\mathrm{n})$ & duty $\min (5 \%)$ & duty max $(11 \%)$ \\
\hline $100,000,000$ & 5,000 & 50 & 100 & 5 & 11 \\
\hline $100,000,000$ & 50,000 & 50 & 1000 & 50 & 110 \\
\hline $100,000,000$ & 500,000 & 50 & 10000 & 500 & 1100 \\
\hline $100,000,000$ & $50,000,000$ & 50 & 1000000 & 50000 & 110000 \\
\hline
\end{tabular}

Table III.3: PWM minimum duty and maximum duty for different period values Multiple instances of the PWM generator were added to the reconfigurable modules depending upon the different PWM signals needed to be generated. The duty parameter of the PWM instances were set from the software. The order of the duty value depended upon the period around which the PWM generator is designed. In this work the PWM initial throttle value is set as a constant in the software which was constantly adjusted using PID algorithm.

\section{III.5 Software Design}

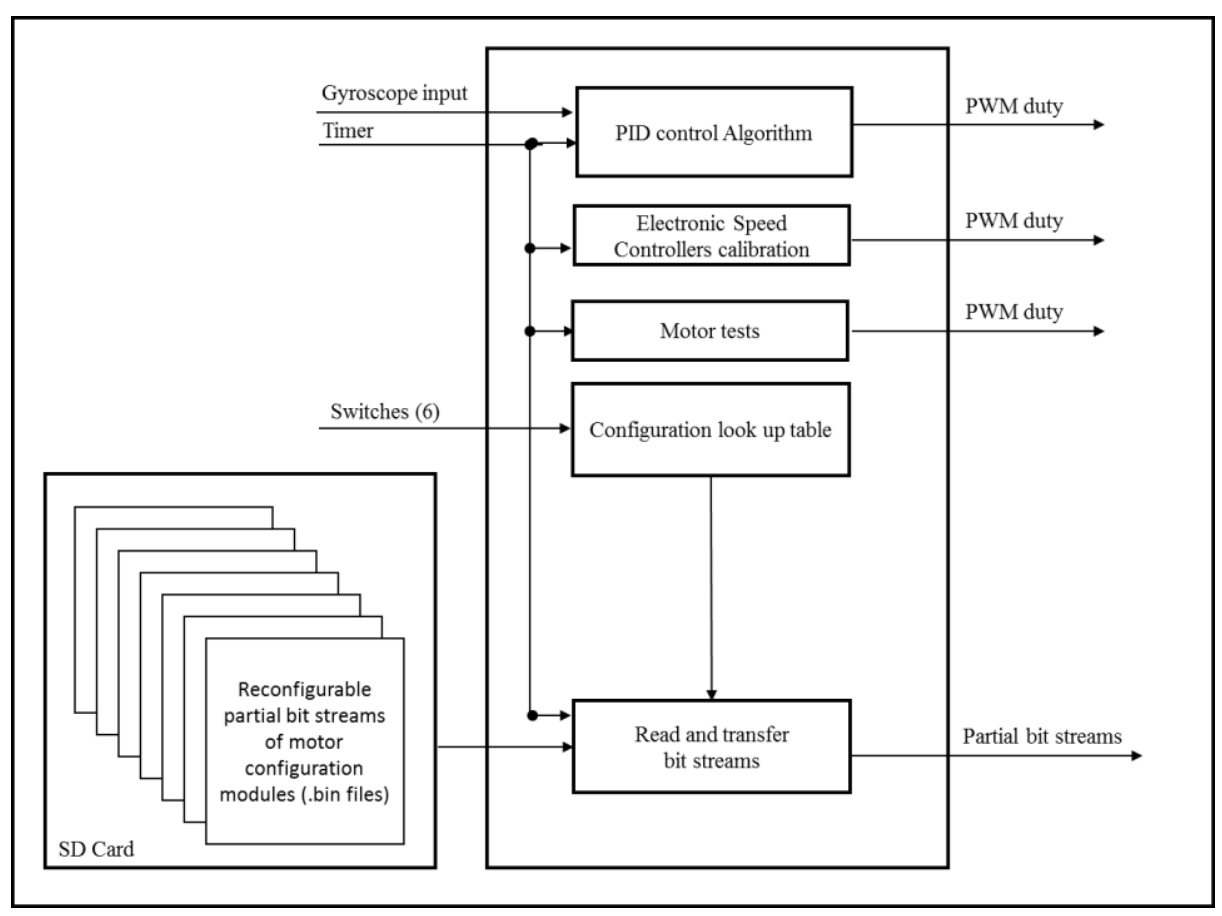

Figure III.15: Software design 
The main functions of the software side of the system was to manage input/output, to implement PID control algorithm, and to manage partial hardware reconfiguration (Figure III.15).

\section{III.5.1 Input/output}

In the scope of this work the objective of the control algorithm is to balance the multirotor on its roll axis. Hence the angular velocity about the $\mathrm{x}$ axis of the gyro sensor was the input for the PID controller. The PID controller calculates error correction values and it is used to modify the PWM duty cycle for the PWM generator. The software also gets input from the slide switches. The input from the six slide switches are decoded into a decimal number. The decimal code is used as an index to look up the output configuration in the look up table implemented as a header file.

\section{III.5.2 PID Control Algorithm}

The PID control algorithm is implemented as a function which takes in the $\mathrm{x}$ axis gyro sensor data and returns error correction values [17]. In this study the tuning of the PID gains Kp, $\mathrm{Kd}$, and $\mathrm{Ki}$ were done experimentally without using a mathematical model. The period parameter in the PID generator was set high at 50,000,000 so that the PID controller could make fine adjustments in the motor speeds in order to balance the multirotor on its roll axis.

\section{III.5.3 Managing Partial Hardware Reconfiguration}

The partial reconfiguration of the reconfigurable partition within the hardware design is managed by the software. All the partial bit streams are stored in an SD card. The software part reads each partial bit stream in the SD card into the RAM. The output configuration codes in the output configuration lookup table are associated with one of the available bit streams. When the input from the slide switches changes, the software will look up the configuration code from the 
lookup table. Then the bit stream associated with the configuration code is sent to the reconfigurable partition and the partition is configured with the new multirotor arm configuration.

In addition the software side has other functions like performing calibration of electronic speed controllers. It is done to set the highest possible PWM duty and lowest PWM duty in the electronic speed controllers. The motors can be also tested individually or as a set from the software side. 


\section{Chapter IV Experiments and Results}

\section{IV.1 Overview}

The adaptive multirotor reconfiguration hardware/software system was tested to study its performance and the relation between various technical parameters of the design. We have mainly three hardware/software designs to experiment with. The experiments and the observations are explained in this chapter.

\section{IV.2 Experimental Setup}

We needed an experimental setup which we can be used to test the performance of our hardware/software multirotor controller (Figure IV.1). Either '+' or ' $\mathrm{X}$ ' frame configuration can be used to test a multirotor [11]. The axis of rotation we chose was the roll axis in the ' $\mathrm{X}$ ' multirotor mode. The reason behind this decision is that in the ' $\mathrm{X}$ ' multirotor mode the arms of the multirotor are distributed on either side of the axis. There are no arms along the axis of rotation. This makes it convenient to test the system in different configurations.

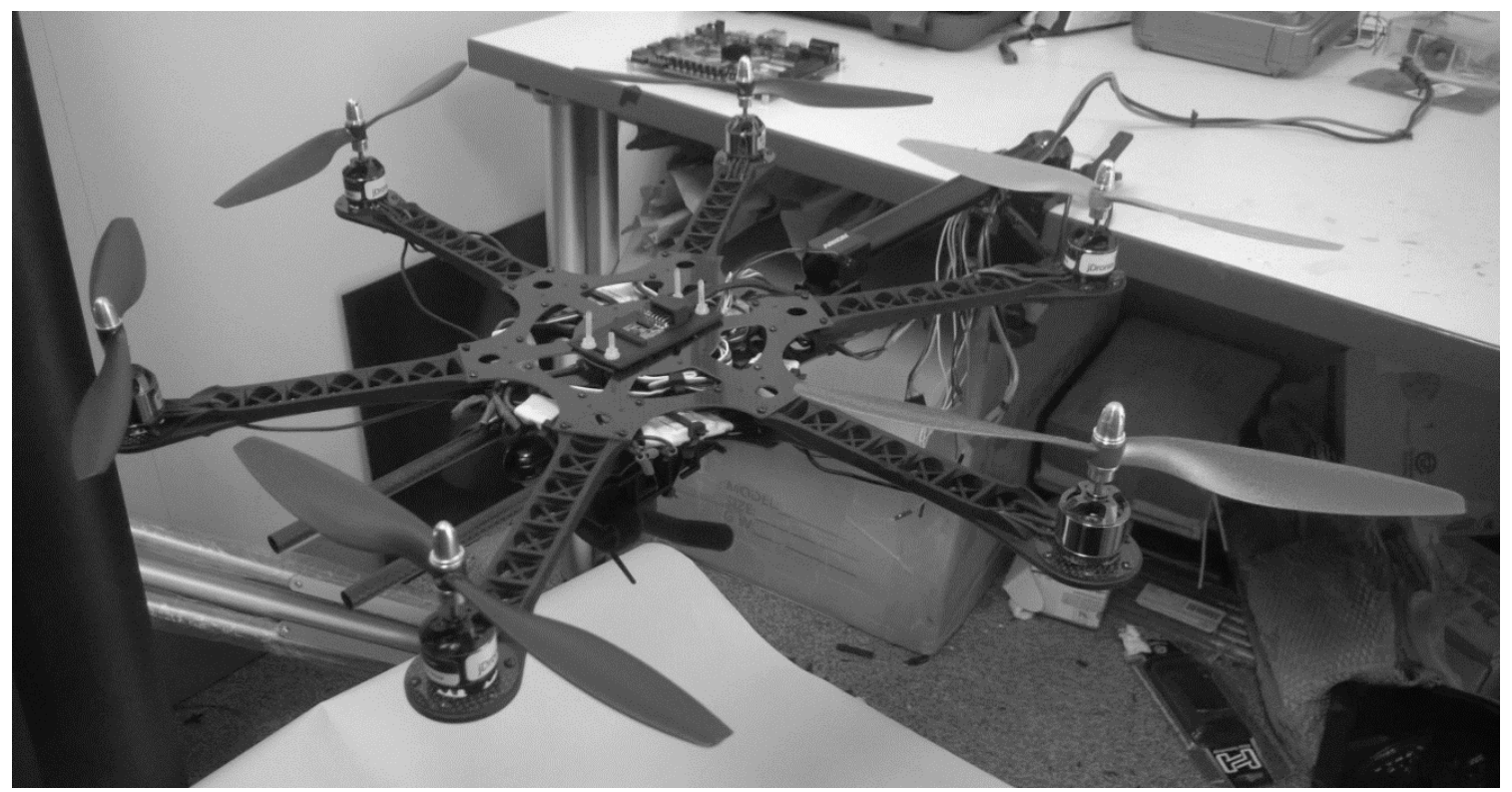

Figure IV.1: Experimental setup (Photo taken by author) 
The multirotor was mounted on an Arkon heavy duty tablet mount. The mount has a C clamp at the base with a ball joint. It has two cylindrical bars joined at a hinge. One of the cylindrical bars is connected to the ball joint. The choice of this mount made it very convenient to position the test piece depending upon the test type (single motor tests, preliminary PID balancing tests and the full system).

All designs were tested on the Xilinx Zynq SoC Prototyping board ZedBoard. In this study only the gyro sensor was placed on the multirotor. The ZedBoard was placed near the multirotor. The PWM signals to the motors and the gyro sensor inputs were connected through wires. A 3 cell, $11.1 \mathrm{~V}, 5000 \mathrm{mAh}$ LiPo battery was used to power the motors. An external power source was used to power the ZedBoard. The multirotor was mounted on the Arkon tablet mount using a 3D printed part. A housing for the gyro sensor was also 3D printed. The parts were modelled in Autodesk 123 Designs and printed on replicator 3G 3D printer.

\section{IV.3 Experiment0: Simple Hardware Design}

In this experiment the simple design of the adaptive multirotor controller implemented without partial hardware reconfiguration was tested. All seven multirotor configurations were implemented on the FPGA fabric as separate hardware blocks. The configurations were connected to the output port through a 7-to-1 multiplexer. The objective of this experiment based on this simple design was to form an understanding of the base case. That is, how the adaptive configuration controller will be implemented on the FPGA without using partial hardware reconfiguration. The gyro sensor hardware IP was not included in the design used in this experiment. In addition the value set for period parameter in the PWM generator was 100. So the PWM duty cycle had only 7 discrete levels from $5 \%$ to $11 \%$. Therefore, this experiment did not have scope for a PID controller implementation in software. As there was no control algorithm 
running on the software side of this design the propellers were not connected to the motors during the tests. The FPGA resources utilization statistics for this design is shown in Table IV.1.

\begin{tabular}{|l|l|l|l|}
\hline \multicolumn{4}{|c|}{ Implemented Utilization } \\
\hline Resource & Utilization & Available & Utilization \\
\hline Register & 351 & 106400 & $1 \%$ \\
\hline LUT & 646 & 53200 & $1 \%$ \\
\hline Slice & 222 & 13300 & $1 \%$ \\
\hline IO & 14 & 200 & $7 \%$ \\
\hline BUFG & 2 & 32 & $6 \%$ \\
\hline
\end{tabular}

Table IV.1: Implemented FPGA resources utilization of Design 0

\section{IV.4 Experiment1: Hardware Design with a Reconfigurable Partition}

In this experiment the adaptive multirotor configuration system which uses runtime partial hardware reconfiguration was used. The control module in the design was implemented as a reconfigurable partition. The seven multirotor configurations were created as partial bit streams. The seven configurations are one six arm configuration, three four arm configurations, two three arm configurations and one configuration where all motors were off. As the control module was implemented as a reconfigurable partition only one of the seven partial bit streams were implemented on the FPGA fabric at a time. The starting configuration of this system was set to be the six arm configuration.

All the seven partial bit streams were stored in an SD card inserted into the SD card slot of the ZedBoard. The partial bit stream associated with that each configuration was transferred into the reconfigurable partition from the RAM as needed.

The main objective of this experiment was to study the difference in FPGA resource utilization compared to the design without partial hardware reconfiguration. Like the previous experiment this experiment did not include the gyro sensor. Different values for the period 
parameter for the PWM generator was tested. There was no implementation of control algorithm on the software side in this design also.

\begin{tabular}{|l|l|l|l|}
\hline \multicolumn{4}{|c|}{ Implemented Utilization } \\
\hline Resource & Utilization & Available & Utilization \\
\hline Register & 209 & 106400 & $1 \%$ \\
\hline LUT & 430 & 53200 & $1 \%$ \\
\hline Slice & 141 & 13300 & $1 \%$ \\
\hline IO & 14 & 200 & $7 \%$ \\
\hline BUFG & 1 & 32 & $3 \%$ \\
\hline
\end{tabular}

Table IV.2: Implemented FPGA resources utilization of Design 1

The size of the reconfigurable partition is set during the implementation of this design during 'PlanAhead tool' design flow. The size of the reconfigurable partition is set in such a way that it meets all requirements of the partial bit streams (reconfigurable modules). Partial hardware reconfiguration does support clock divider logic in a reconfigurable partition. Hence the clock divider logic is a part of the static logic.

Compared to the FPGA resource utilization statistics of the first design (Table IV.1) which does not have partial hardware reconfiguration, this design uses less resources (Table IV.2). This is because only one control module is physically present on the FPGA fabric at a time. In our experiments each control module is designed to perform the simple function of generating and distributing PWM signals to motors. Therefore each control module takes up only a tiny amount of FPGA resources. Hence, the FPGA resource utilization data of the two experiments do not show a huge difference. More space will be saved as each individual control module gets bigger when more functions are added in the future developments. The space saved in this design can be used for adding additional on-board features as part of future work. 


\section{IV.5 Experiment2: Prototype with PID control}

The objective of this experiment was to test the performance of the system in a practical application. Our system was used to balance a multirotor on its roll axis. In addition to the control module implemented as a reconfigurable partition, a gyro sensor was added to the hardware part of the system. A PID controller was implemented in the software part. In this experiment the multirotor reconfiguration part was the same as the previous experiment. The reconfiguration of the reconfiguration partition was triggered by the 6 bit input from the slide switches. The bit streams stored in the SD card were transferred into the RAM and then transferred to the reconfigurable partition as needed.

The software part constantly read in the slide switch input as well as the gyro sensor input. As the $\mathrm{x}$ axis of the gyro sensor was the axis about which the multirotor could move in the experimental setup only the $\mathrm{x}$ axis value was passed to PID function. The PID function calculated error correction term using standard PID algorithm. Then the PWM duty cycle values for the motors were adjusted constantly using the error correction term from the PID function. The FPGA resource utilization statistics for this experiment is shown in Table IV.3.

\begin{tabular}{|l|l|l|l|}
\hline \multicolumn{4}{|c|}{ Implemented Utilization } \\
\hline Resource & Utilization & Available & Utilization \\
\hline Register & 825 & 106400 & $1 \%$ \\
\hline LUT & 1227 & 53200 & $2 \%$ \\
\hline Slice & 434 & 13300 & $3 \%$ \\
\hline IO & 26 & 200 & $13 \%$ \\
\hline BUFG & 1 & 32 & $3 \%$ \\
\hline
\end{tabular}

Table IV.3: Implemented FPGA resources utilization of Design 2

The FPGA resource utilization of this is design is higher than the other two designs. It is because additional FPGA resources are utilized to implement the gyro sensor IP as a part of the static (fixed) logic. This design also uses some hardware resources to interface the slide switches. 
The reconfigurable partition for the control modules is implemented within a FPGA block with the same size as that of the previous experiment. This was ensured by marking the partition manually during the design process.

\section{IV.6 Observations}

\section{IV.6.1 PWM vs Current}

The current drawn from the battery by a single multirotor motor was monitored using the AttoPilot Voltage and Current Sensor. The relation between the PWM duty and the current drawn is shown in Table IV.4.

\begin{tabular}{|c|c|c|c|c|c|c|c|c|c|c|}
\hline \multicolumn{11}{|c|}{ PWM duty vs Current } \\
\hline & \multicolumn{10}{|c|}{ current $(\mathrm{A})$} \\
\hline PWM duty $(\%)$ & $\mathrm{V}=12.16$ & $\mathrm{~V}=11.9$ & $\mathrm{~V}=11.86$ & $\mathrm{~V}=11.78$ & $\mathrm{~V}=11.7$ & $\mathrm{~V}=12.32$ & $\mathrm{~V}=12.09$ & $\mathrm{~V}=11.93$ & $\mathrm{~V}=11.86$ & $\mathrm{~V}=11.7$ \\
\hline 5 & 0 & 0 & 0 & 0 & 0 & 0 & 0 & 0 & 0 & 0 \\
\hline 5.5 & 0 & 0 & 0 & 0 & 0 & 0 & 0 & 0 & 0 & 0 \\
\hline 6 & 0 & 0 & 0 & 0 & 0 & 0 & 0 & 0 & 0 & 0 \\
\hline 6.5 & 0.54 & 0 & 0 & 0 & 0.54 & 0 & 0 & 0.27 & 0.27 & 0.54 \\
\hline 7 & 1.08 & 0.81 & 0.81 & 0.81 & 0.81 & 0 & 0 & 1.08 & 0.81 & 1.08 \\
\hline 7.5 & 1.62 & 1.35 & 1.35 & 1.35 & 1.35 & 0.54 & 1.62 & 1.62 & 1.62 & 1.89 \\
\hline 8 & 3.24 & 2.7 & 2.43 & 2.43 & 2.43 & 1.89 & 2.97 & 2.7 & 2.7 & 2.97 \\
\hline 8.5 & 4.59 & 4.05 & 4.05 & 4.05 & 3.78 & 3.24 & 4.32 & 4.32 & 4.32 & 4.59 \\
\hline 9 & 6.49 & 5.95 & 5.68 & 5.41 & 5.41 & 5.41 & 6.22 & 5.95 & 5.68 & 5.95 \\
\hline 9.5 & 8.38 & 7.84 & 7.57 & 7.57 & 7.3 & 7.03 & 8.11 & 7.84 & 7.57 & 7.84 \\
\hline 10 & 9.73 & 9.46 & 9.46 & 9.19 & 9.19 & 8.65 & 9.73 & 9.73 & 9.46 & 9.46 \\
\hline 10.5 & 9.73 & 9.46 & 9.46 & 9.19 & 9.19 & 8.38 & 9.73 & 9.73 & 9.46 & 9.46 \\
\hline 11 & 9.73 & 9.46 & 9.46 & 9.19 & 9.19 & 8.38 & 9.73 & 9.73 & 9.46 & 9.46 \\
\hline
\end{tabular}

Table IV.4: Current drawn at different PWM duty (higher the PWM higher the motor speed)

The PWM duty value was varied from 5\% to $11 \%$ through $0.5 \%$ steps. The voltage measured in each run is recorded in the top row of the Table IV.4. The measured current values were plotted against PWM duty values. The graph (Figure IV.2) showed an almost linear relationship between the PWM duty and the current drawn. 


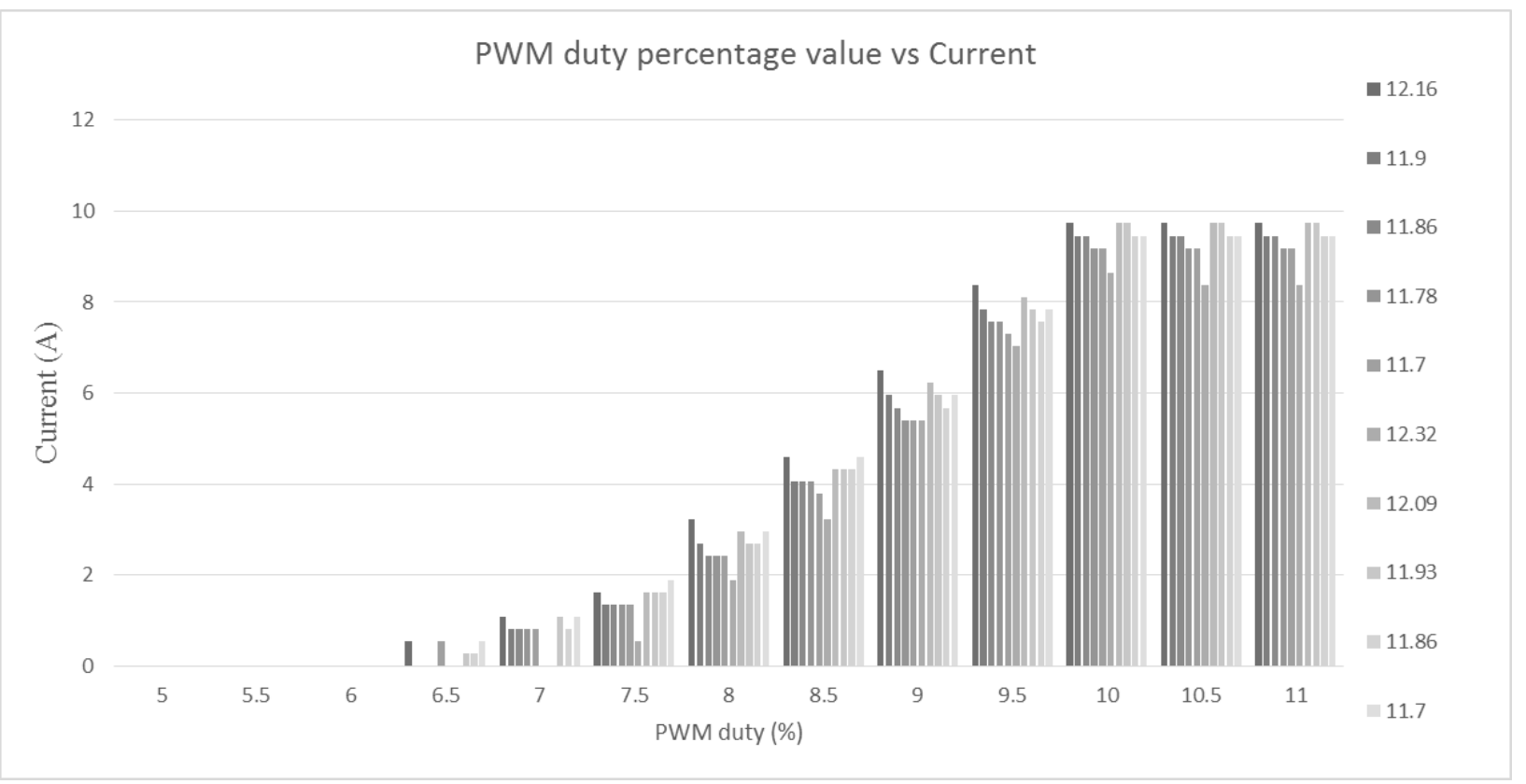

Figure IV.2: PWM duty vs Current (at different values of Voltage)

\section{IV.6.2 Multirotor Configurations vs Current}

The current drawn from the battery by each of the multirotor arm configurations was measured using the AttoPilot Voltage and Current Sensor. The relation between the multirotor arm configurations and the current drawn is shown in Table IV.5. All readings were recorded at a PWM value of $10 \%$ (bottom limit of PWM is 5\% and top limit is $11 \%$ ).

\begin{tabular}{|c|c|c|c|c|c|c|c|c|c|c|c|}
\hline \multicolumn{12}{|c|}{ Multirotor Configurations vs Current } \\
\hline & & \multicolumn{10}{|c|}{ current drawn (A) } \\
\hline \multicolumn{2}{|c|}{ PWM duty } & \multicolumn{10}{|c|}{$10 \%$} \\
\hline \multicolumn{2}{|c|}{ measured voltage $(\mathrm{V})$} & 12.09 & 11.78 & 11.39 & 12.24 & 11.7 & 11.47 & 11.24 & 12.24 & 11.01 & 11.39 \\
\hline \multirow{6}{*}{$\begin{array}{c}\text { Multirotor } \\
\text { Configurations }\end{array}$} & 6 & 50.27 & 48.65 & 46.49 & 52.43 & 47.57 & 46.49 & 45.41 & 51.62 & 47.03 & 45.41 \\
\hline & $4(1,3,4,6)$ & 34.32 & 32.97 & 31.89 & 34.86 & 32.97 & 31.89 & 31.08 & 34.86 & 32.7 & 31.62 \\
\hline & $4(1,2,4,5)$ & 34.32 & 33.51 & 32.7 & 35.41 & 33.51 & 32.43 & 30.81 & 35.41 & 32.24 & 31.89 \\
\hline & $4(2,3,5,6)$ & 34.32 & 33.24 & 32.43 & 34.32 & 32.97 & 31.89 & 31.08 & 34.59 & 32.43 & 31.62 \\
\hline & $3(1,3,5)$ & 27.3 & 25.14 & 24.05 & 27.84 & 26.22 & 24.78 & 23.51 & 27.84 & 25.68 & 24.86 \\
\hline & $3(2,4,6)$ & 26.49 & 25.68 & 25.14 & 27.03 & 25.41 & 24.86 & 24.32 & 23.51 & 25.14 & 24.59 \\
\hline
\end{tabular}

Table IV.5: Current drawn for different configurations (more motor draw more current)

The measured values were plotted as clustered columns (Figure IV.3). As expected the six motor configuration draws maximum current and less current is drawn by configurations with lower number of motors. 


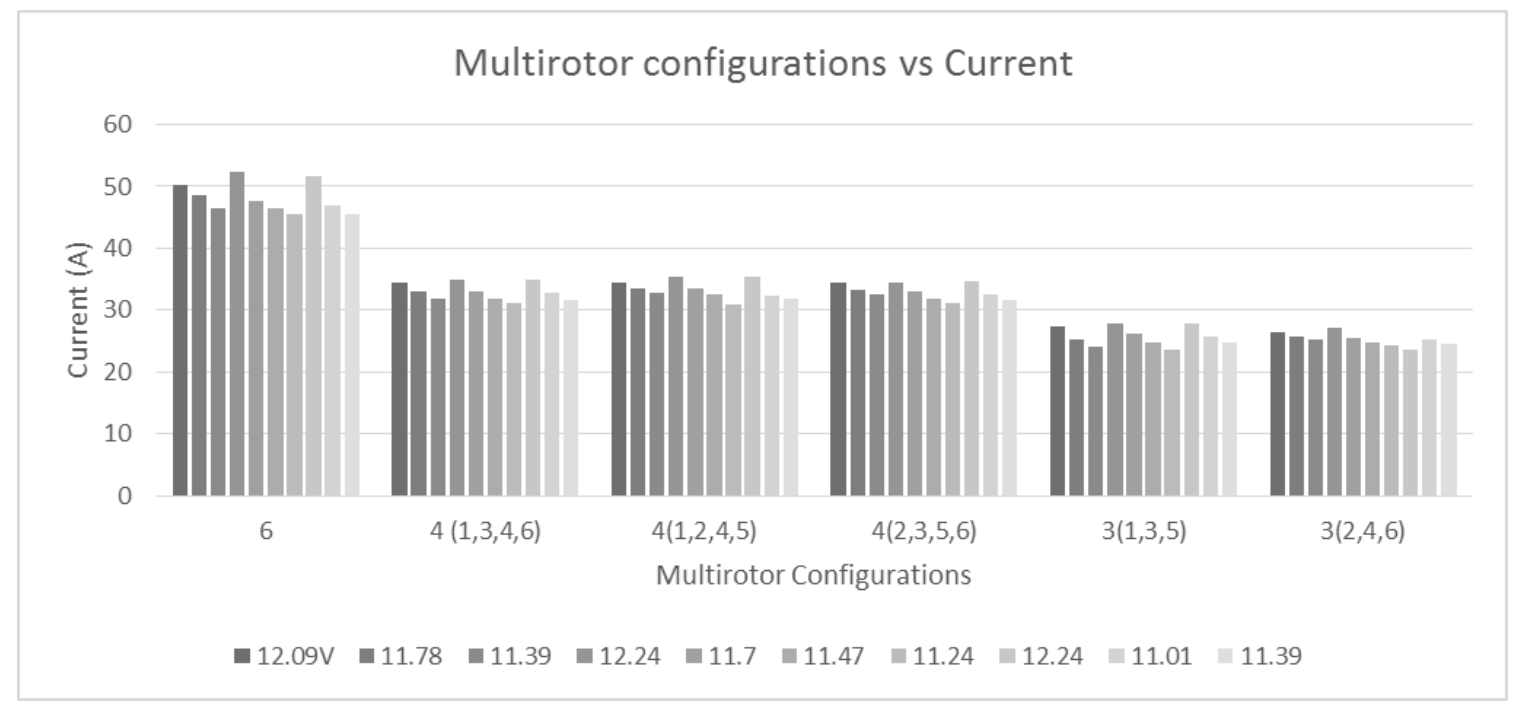

Figure IV.3: Multirotor configuration vs Current (at different values of Voltage)

All current and voltage measurements were made using Sparkfun AttoPilot Voltage and Current Sense Breakout - 180A. The values were read out from a serial monitor. The measured values had a precision of two digits $(0.00 \mathrm{~A})$. The power source used was a 3 cell, $11.1 \mathrm{~V}$, 5000mAh LiPo battery. As the voltage was calculated based on the input from the Arduino microcontroller analog input pin, there was slight variation in the reading when the experiments were repeated. Hence, the readings were taken from the experiments ten times each as shown in the in the tables (Table IV.4 and Table IV.5). 


\section{Chapter V Conclusion and Future Work}

\section{V.1 Conclusion}

In this study a hardware/software adaptive multirotor controller platform was designed and developed. The design was targeted on the processing system and FPGA fabric combination of Xilinx Zynq System on Chip. FPGA runtime partial reconfigurations technology was used to make the platform adapt different multirotor arm configurations. The design was tested on a ZedBoard, the Zynq System on Chip prototyping board. The FPGA resource utilization of different designs were recorded. A PWM signal generator was implemented as a hardware module to drive the motors. The current drawn by a motor with respect to the PWM duty cycle was recorded. PID control algorithm was implemented in software to perform single axis balancing of a various arm configurations of the multirotor. The multirotor arm configuration adaptivity was tested along with the PID algorithm.

The multirotor used for tests (XAircraft DIY HEXA) had six motors arranged in a conventional hexacopter configuration. Hence the yaw angular momentum was balanced only for the multirotor arm configuration where all six arms were used. For other configurations the yaw angular momentum was not zero most of the time. It did not affect the single axis balancing to a great extent as the multirotor was mounted without freedom to turn on its yaw axis. In future developments a possible solution to balance the net yaw angular momentum of any multirotor arm configuration is to use two motors with opposite spin on each arm. One motor should point up and one motor should point down. The net angular momentum of each arm should be then balanced. Hence, a combination of any number of multirotor arms should keep the net yaw angular momentum balanced. 
The adaptive multirotor platform was developed with a three phase design process. First, as a base case an adaptive multirotor platform was designed without using partial hardware reconfiguration technology. The seven different multirotor arm configurations were added as hardware sub-modules. This design gave us an understanding of the working of a simple adaptive platform which can switch between different multirotor arm configurations. Then FPGA runtime partial reconfiguration technology was added to the design. Therefore hardware implementation of one of the seven multirotor arm configurations had to be configured in the FPGA at a time. The hardware modules for the seven different configurations were stored as partial bit streams on the SD card. The partial bit streams were transferred into the reconfigurable hardware partition as needed. In the final design, hardware modules for interfacing gyro sensor to the existing system was added. PID control algorithm was implemented on the software side to perform single axis balancing of the multirotor.

The runtime partial reconfiguration of multirotor control modules was tested successfully. In this study the primary function of control modules was PWM generation depending upon duty percentage values set from the software. Hence, the time taken to switch the system from one configuration to other was negligible. The switching process has to be optimized to ensure safety of equipment. It needs more research to implement an efficient switching protocol. The single axis balancing of the multirotor with runtime switching of multirotor arm configurations was not perfect. This is because of imperfections in the experimental setup and because of signal noise in the gyro sensor due to vibration. In addition the PID constants were tuned without mathematically modelling the multirotor controller. The experimental setup has to be improved for better results in the future. Mathematical modelling of the system is also need to be developed for implementing an effective adaptive controller. 


\section{V.2 Potential Applications}

The adaptive multirotor controller platform has many potential applications. It needs to be studied thoroughly to understand its strengths and limitations. An application of this type of controller will be multirotors used for object delivery and retrieval [7]. If this technology is developed further the arm configuration of the multirotor can be switched depending upon the presence and absence of a load.

One hypothetical application for this study is autonomous configuration adaptivity. It was not tested experimentally as part of this study. However, we believe this might be one possible future application for this study. It is briefly described below.

We know the relation between the type of multirotor arm configurations and the maximum current drawn from the battery (Table IV.5). Assume that we are using a $5000 \mathrm{mAh}$ battery. It means that theoretically the battery can discharge 5 Amperes for 60 minutes. Depending upon this we can theoretically estimate the operational duration of a multirotor arm configuration. If a configuration draws 50 Amperes of current, theoretically the battery can power it for 10 minutes. Taking the average current value for each configuration from the table we can theoretically estimate average operational time for each configuration. Table V.1 is constructed based on an assumption that the battery is operated for the time duration listed. However, the most of electronic speed controllers used in multirotors will cut off the battery if its voltage drops below the limit for safe operation.

\begin{tabular}{|c|c|c|c|}
\hline MultirotorConfigurations & Average Current $(\mathrm{A})$ & Battery rating (mAh) & Estimated duration of operation (minutes) \\
\hline 6 & 48.137 & 5000 & 6.23 \\
\hline $4(1,3,4,6)$ & 32.916 & 5000 & 9.11 \\
\hline $4(1,2,4,5)$ & 33.223 & 5000 & 9.03 \\
\hline $4(2,3,5,6)$ & 32.889 & 5000 & 9.12 \\
\hline $3(1,3,5)$ & 25.722 & 5000 & 11.66 \\
\hline $3(2,4,6)$ & 25.217 & 5000 & 11.90 \\
\hline
\end{tabular}

Table V.1: Theoretical estimation of operation time (within the context of our hypothetical example) 
Now assume that a single motor produces enough thrust to carry a load of ' $\mathrm{W}$ ' units. Then the three arm configuration will carry $3 \mathrm{~W}$ units, four arm configuration can support $4 \mathrm{~W}$ units, and the six arm configuration can carry $6 \mathrm{~W}$ units. It should be noted that it is assumed that the load also includes the weight of the multirotor.

Using the Table V.1 and the assumption of the weights, we can construct the following table. This table is constructed based on a broad assumption that as the currents were measured at $10 \%$ duty cycle it can be considered as the worst case current measurement.

\begin{tabular}{|c|c|c|c|c|c|c|}
\hline \multicolumn{7}{|c|}{ using load to decide configuration based on filight duration at highest PWM duty } \\
\hline & \multicolumn{7}{|c|}{ MultirotorConfigurations } \\
\hline Load (units) & 6 & $4(1,3,4,6)$ & $4(1,2,4,5)$ & $4(2,3,5,6)$ & $3(1,3,5)$ & $3(2,4,6)$ \\
\hline 3W & 6.23 & 9.11 & 9.03 & 9.12 & 11.66 & 11.90 \\
\hline 4W & 6.23 & 9.11 & 9.03 & 9.12 & $X$ & $X$ \\
\hline 6W & 6.23 & $\mathrm{X}$ & $\mathrm{X}$ & $\mathrm{X}$ & $\mathrm{X}$ & $\mathrm{X}$ \\
\hline
\end{tabular}

Table V.2: Hypothetical application: table showing operation duration for different configurations for a given load

Table V.2 is an abstract representation of a possible lookup table to be implemented to facilitate autonomous configuration adaptivity. By developing a table like this through rigorous experimental study, given the weight of the load a multirotor should be able to choose the best configuration to meet a target time/performance. This is a simplistic example which considers only the capacity of the power source and the load. However, better estimations could be made by conducting a detailed study [14].

Ability to switch configurations based on environmental factors like wind can be another potential application for this work. In addition, another application can be a multirotor that can use the best configuration depending upon the nature of flight (takeoff/land, hover, cruise etc). 


\section{V.3 Future work}

This work is in its initial state. There are opportunities to improve the current system as well as to add more functionalities to the system. Three ways to improve the current system are briefly explained below.

\section{V.3.1 Multiplexed Dual Reconfigurable Partitions}

In the current implementation of the adaptive multirotor controller there is only one reconfigurable partition. The reconfigurable partition is where various control modules are configured. When the multirotor needs to change its current arm configuration, it reconfigures the reconfigurable partition using the new partial bit stream. There is a slight delay in this process. This delay will increase in the future as the reconfiguration modules become bigger in size when more functionality is moved to the hardware side from the software side. The multirotor controller will be in a floating state during the reconfiguration. Hence, we need to redefine the reconfiguration process to avoid the floating state.

One potential solution is the use of two identical reconfigurable partitions under the control of a supervisor module [22]. The two reconfigurable partitions can be configured as control modules using partial bit streams. However, only one of the reconfigurable partitions will be active at a time. When the multirotor need to change its configuration the reconfigurable module which is currently inactive is configured with the new partial bit stream. Once the reconfiguration is complete and the reconfigurable partition is ready, the supervisor will make the reconfigurable partition active and the other one is made inactive. The new configuration takes effect only when the partial reconfiguration is complete and the reconfigurable partition is ready. Hence the occurrence of a floating state can be prevented by implementing two identical reconfigurable partitions controlled by a supervisor. 


\section{V.3.2 User Input from a Radio/Bluetooth Controller}

One of the key functions that needs to be implemented on the system is user input processing. Radio controllers are a popular multirotor control device. A Bluetooth based controller will be also effective for low range indoor experimental work. A Bluetooth based controller will be cheaper compared to a radio transmitter. A simple user interface can be developed as a smart phone application. In addition a Bluetooth based controller will help us setup software control interfaces (apps) on multiple mobile devices. The Bluetooth peripheral modification module from Digilent Inc will be ideal for implementing a Bluetooth controller.

An ideal controller for the adaptive controller should have at least five channels. Four channels to be used to control throttle, roll, pitch, and yaw of the multirotor. The fifth channel can be used as a control input to trigger multirotor arm reconfiguration. The fifth channel can have multiple discrete signal levels to select different multirotor arm configurations.

\section{V.3.3 Mathematical Modeling of Multirotor}

Developing a mathematical model of the multirotor system under development will be necessary to implement a PID control algorithm effectively. The future version of the multirotor under development can have up to 12 motors. It is necessary to have an accurate understanding of the dynamics of the multirotor. Adjustments on the software side such as tuning of PID control algorithm, calculating offsets for input/output values etc. can be done better with a mathematical model. In addition, control modules for different multirotor parameters like pitch, yaw, altitude etc. are also needed. Further development also need to incorporate an inertial measurement unit (IMU) which combines a gyroscope and an accelerometer. The adaptive controller system has to go through multiple design iterations before it can takeoff, hover, cruise and land safely. 


\section{Bibliography}

1. d'Oleire-Oltmanns, S., Marzolff, I., Peter, K., \& Ries, J. (2012). Unmanned Aerial Vehicle (UAV) for Monitoring Soil Erosion in Morocco. Remote Sensing, 4(12), 33903416. doi:10.3390/rs4113390

2. Learn.sparkfun.com,. (2015). Motors and Selecting the Right One - learn.sparkfun.com. Retrieved 6 July 2015, from https://learn.sparkfun.com/tutorials/motors-and-selectingthe-right-one?_ga=1.34767073.1527219636.1432137882

3. Learn.sparkfun.com,. (2015). Battery Technologies - learn.sparkfun.com. Retrieved 6 July 2015, from https://learn.sparkfun.com/tutorials/batterytechnologies?_ga=1.235593793.1527219636.1432137882\%23lithium-polymer

4. Phenoxlab.com,. (2015). Phenox | Phenox Lab. Retrieved 6 July 2015, from http://phenoxlab.com/?page_id=296

5. Fogelberg, J. (2013). Navigation and Autonomous Control of a Hexacopter in Indoor Environments (Msc). Lund University.

6. Chaimowicz, L., \& Kumar, V. (2007). Aerial Shepherds: Coordination among UAVs and Swarms of Robots. Distributed Autonomous Robotic Systems 6, 243-252. doi:10.1007/978-4-431-35873-2_24

7. Amazon.com,. (2015). Amazon Prime Air. Retrieved 6 July 2015, from http://www.amazon.com/b?node=8037720011

8. E-volo.com,. (2015). e-volo. Retrieved 5 July 2015, from http://www.e-volo.com/

9. Hover-bike.com,. (2015). MA Hoverbike. Retrieved 5 July 2015, from http://www.hoverbike.com/MA/

10. Maxfield, C. (2008). FPGAs: Instant Access. Amsterdam: Newnes.

11. Norris, D. (2014). Build your own quadcopter. New York: McGraw-Hill Education.

12. Crockett, L., Elliot, R., Enderwitz, M., \& Stewart, R. (2014). The Zynq Book: Embedded Processing with the ARM Cortex-A9 on the Xilinx Zynq-7000 all Programmable SoC. Strathclyde Academic Media.

13. Partial Reconfiguration User Guide (14.5). (2013) (14th ed.). Retrieved from http://www.xilinx.com/support/documentation/sw_manuals/xilinx14_7/ug702.pdf

14. D'Andrea, R. (2014). Guest Editorial Can Drones Deliver?. IEEE Trans. Automat. Sci. Eng., 11(3), 647-648. doi:10.1109/tase.2014.2326952 
15. T G Zhou, T., T X Zhou, D., \& H B Zhou, A. (2014). Unmanned drone, robot system for delivering mail, goods, humanoid security, crisis negotiation, mobile payments, smart humanoid mailbox and wearable personal exoskeleton heavy load flying machine. USA.

16. Grc.nasa.gov,. (2015). Aircraft Rotations. Retrieved 6 July 2015, from https://www.grc.nasa.gov/www/k-12/airplane/rotations.html

17. Lewis, J. (2014). Feedback Control Systems Demystified: Volume 1 Designing PID Controllers. Surber Press.

18. ZedBoard (Zynq ${ }^{\mathrm{TM}}$ Evaluation and Development) Hardware User's Guide. (2012) (1st ed.). Retrieved from http://zedboard.org/sites/default/files/ZedBoard_HW_UG_v1_1.pdf

19. Delahaye, J., Gogniat, G., Roland, C., \& Bomel, P. (2004). Software Radio and Dynamic Reconfiguration on a DSP/FPGA platform. Frequenz, 58(5-6). doi:10.1515/freq.2004.58.5-6.152

20. Lackey, R., \& Upmal, D. (1995). Speakeasy: the military software radio. IEEE Communications Magazine, 33(5), 56-61. doi:10.1109/35.392998

21. Bhandari, S., Subbaraman, S., \& Pujari, S. (2010). Power Reduction in Embedded System on FPGA Using on the Fly Partial Reconfiguration. 2010 International Symposium On Electronic System Design. doi:10.1109/ised.2010.23

22. Danne, K., Bobda, C., \& Kalte, H. (2003). Increasing Efficiency by Partial Hardware Reconfiguration: Case Study of a Multi-Controller System. In International Conference on Engineering of Reconfigurable Systems and Algorithms. Las Vegas, Nevada, USA.

23. Ganesh Nayak, N. (2013). Accelerated Computation using Runtime Partial Reconfiguration (Master's). University of Stuttgart.

24. Digilentinc.com,. (2015). Digilent Inc. - Digital Design Engineer's Source. Retrieved 6 July 2015, from https://www.digilentinc.com/Products/Detail.cfm?NavPath=2,401,937\&Prod=PMODGYRO

25. Compact DC Voltage and Current Sense PCB with Analog Output. (2011) (1st ed.). Retrieved from http://cdn.sparkfun.com/datasheets/Sensors/Current/DC\%20Voltage\%20and\%20Current $\% 20$ Sense $\% 20 \mathrm{PCB} \% 20$ with\%20Analog\%20Output.pdf

26. Haskell, R., \& Hanna, D. (2009). Digital design using digilent FPGA boards (2nd ed., pp. 185-190). Rochester Hills, MI: Learning by Example. 